\title{
A Drosophila Su(H) Model of Adams-Oliver Syndrome Reveals Notch Cofactor Titration as a Mechanism Underlying Developmental Defects
}

Ellen K. Gagliani ${ }^{1,9}$, Lisa M. Gutzwiller ${ }^{3,9}$, Yi Kuang ${ }^{4}$, Yoshinobu Odaka ${ }^{5}$, Phillipp Hoffmeister ${ }^{6}$, Stefanie Hauff $^{6}$, Aleksandra Turkiewicz ${ }^{8}$, Emily Harding-Theobald ${ }^{7}$, Patrick J. Dolph ${ }^{7}$, Tilman Borggrefe ${ }^{8}$, Franz Oswald $^{6}$, Brian Gebelein ${ }^{2,3^{*}}$, and Rhett A. Kovall ${ }^{*}$

${ }^{1}$ Department of Molecular Genetics, Biochemistry and Microbiology, University of Cincinnati College of Medicine, Cincinnati, OH 45267, USA

${ }^{2}$ Department of Pediatrics, University of Cincinnati College of Medicine, Cincinnati, OH 45229, USA.

${ }^{3}$ Division of Developmental Biology, Cincinnati Children's Hospital Medical Center, 3333 Burnet Ave, MLC 7007, Cincinnati, OH 45229, USA

${ }^{4}$ Graduate program in Molecular and Developmental Biology, Cincinnati Children's Hospital Research Foundation, Cincinnati, OH 45229, USA

${ }^{5}$ Biology Department, University of Cincinnati Blue Ash College, OH 45236, USA

6University Medical Center Ulm, Center for Internal Medicine, Department of Internal Medicine 1, Albert-

Einstein-Allee 23, 89081 Ulm, Germany

${ }^{7}$ Department of Biology, Dartmouth College, Hanover, New Hampshire 03755,

${ }^{8}$ Institute of Biochemistry, University of Giessen, 35392 Giessen, Germany

${ }^{9}$ These authors contributed equally

"Correspondence: kovallra@ucmail.uc.edu (R.A.K.) and Brian.Gebelein@cchmc.org (B.G.) 


\section{ABSTRACT}

Notch signaling is a conserved pathway that converts extracellular receptor-ligand interactions into changes in gene expression via a single transcription factor (CBF1/RBPJ in mammals; $\mathrm{Su}(\mathrm{H})$ in Drosophila). In humans, RBPJ variants have been linked to Adams-Oliver syndrome (AOS), a rare autosomal dominant disorder characterized by scalp, cranium, and limb defects. Here, we found that a previously described Drosophila $\mathrm{Su}(H)$ allele encodes a missense mutation that alters an analogous residue found in an AOS-associated RBPJ variant. Importantly, genetic studies support a model that Drosophila with a single copy of the AOS-like $\mathrm{Su}(\mathrm{H})$ allele behave in an opposing manner as flies with a $S u(H)$ null allele due to a dominant activity of sequestering either the Notch co-activator or the antagonistic Hairless co-repressor. Consistent with this model, AOS-like $\mathrm{Su}(\mathrm{H})$ and Rbpj variants decrease DNA binding activity compared to wild type proteins, but these variants do not significantly alter protein binding to the Notch co-activator or the fly and mammalian co-repressors, respectively. Taken together, these data suggest a cofactor sequestration mechanism underlies AOS phenotypes associated with RBPJ variants, whereby a single RBPJ allele encodes a protein with compromised DNA binding activity that retains cofactor binding, resulting in Notch target gene dysregulation. 


\section{INTRODUCTION}

Notch signaling is a highly conserved pathway that mediates cell-to-cell communication in all metazoans ${ }^{1-3}$. During embryogenesis, Notch plays a crucial role in vasculogenesis, hematopoiesis, neurogenesis, and cardiac development ${ }^{4}$. Additionally, Notch regulates tissue homeostasis, including epidermal differentiation and maintenance, lymphocyte differentiation, muscle and bone regeneration, and angiogenesis ${ }^{4}$. Intriguingly, Notch regulates these diverse processes using a common molecular cascade $^{5}$ (Fig 1A). Signaling is activated when Notch receptors on a cell interact with DSL (Delta, Serrate, Lag-2 for mammalian, Drosophila, and C. elegans orthologs, respectively) ligands on a neighboring cell. In mammals there are four Notch receptors (Notch1-4) and five DSL ligands (DII1,3,4 and Jag1,2), whereas Drosophila have one Notch receptor and two ligands (Delta and Serrate). The Notch-DSL interaction triggers DSL endocytosis, resulting in force induced Notch cleavage ${ }^{6}$, which is mediated by ADAM10 and the $\gamma$-secretase complex. Once cleaved, the Notch intracellular domain (NICD) is freed from the cell membrane, transits to the nucleus, and forms a complex with the transcription factor CSL (CBF1/RBPJ, Su(H) and Lag-1 for mammalian, Drosophila, and C. elegans orthologs, respectively) and the co-activator Mastermind (MAM) ${ }^{7}$. The NICD/CSL/MAM (NCM) complex binds to enhancer and promoter DNA elements to activate gene expression ${ }^{8}$. However, CSL can also function as a transcriptional repressor by forming complexes with co-repressor proteins, such as SHARP and Hairless in mammals and Drosophila, respectively ${ }^{9-11}$. Hence, Notch signal strength in a cell is largely defined by the balance of NCM activating complexes and CSL/co-repressor complexes that regulate target gene expression.

Genetic studies have shown that a subset of Notch-dependent processes are highly sensitive to gene dose. The term Notch derives its name from the original notched wing phenotype identified in Drosophila, resulting from Notch receptor haploinsufficiency. Heterozygous Notch phenotypes are also observable in the number and spacing of bristles on the fly notum. Moreover, human birth defects and developmental syndromes have been linked to haploinsufficiency for three out of the four Notch receptors (NOTCH1/2/3), two out of five ligands (DLL4 and JAG1), and the RBPJ transcription factor 


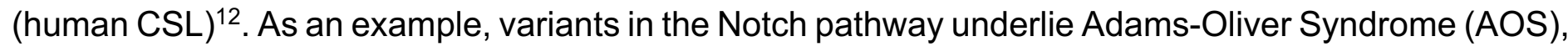

a rare disease defined by scalp aplasia cutis congenita (missing skin and skull tissue) and limb malformations. Additionally, AOS patients can suffer cardiovascular abnormalities, such as cutis marmorata telangiectatica (dilated surface blood vessels), hypertension, and heart defects ${ }^{15-17}$. Genetic studies have revealed that $\sim 40 \%$ of AOS patients inherit dominant mutations within the NOTCH1 receptor, the DLL4 ligand, or the RBPJ transcription factor. In contrast, haploinsufficiency mutations in the NOTCH2 receptor and JAG1 ligand are associated with Alagille Syndrome, a disease characterized by liver, eye, kidney, heart, skeleton, and vasculature defects ${ }^{13}$. Hence, genetic variants within the Notch signaling pathway can cause developmental defects in organisms from flies to humans.

In 2012, Hassad et. al. reported two autosomal dominant variants in RBPJ in separate families with $\mathrm{AOS}^{14}$. Interestingly, this study was the first to report germline variants in RBPJ that cause a disease in humans. These variants (E63G \& K169E) mapped to the DNA binding region of RBPJ (Fig 1C, D), and the authors used cell extracts to show each variant impaired DNA binding ${ }^{14}$. Hence, these variants were classified as loss-of-function alleles. Here, we show that a previously identified allele in $\mathrm{Su}(H)$ (Drosophila CSL), Su(H) ${ }^{\mathrm{T}}$, encodes an E137V mutation at the same highly conserved glutamic acid residue (human RBPJ E63G) as seen in AOS patients. However, prior genetic studies showed that $\mathrm{Su}(H)^{\top 4}$ has "gain-of-function" activity in Drosophila as opposed to the predicted loss-of-function activity suggested for the RBPJ E63G allele ${ }^{15}$. To better explain the discrepancy between these conflicting biochemical and genetic results, we used a combination of quantitative Drosophila genetic studies, DNA binding assays, and protein-protein interaction assays. Our data show that the AOS-like $\mathrm{Su}(H)$ allele and a $\mathrm{Su}(H)$ null allele behave in a largely opposing manner, as the $\mathrm{Su}(H)$ AOS-like allele exacerbated both Notch and Hairless phenotypes, whereas the $S u(H)$ null allele partially suppressed Notch and Hairless phenotypes. Moreover, we found that the $S u(H)$ alleles and the mammalian RBPJ AOS variant alleles encode proteins defective in DNA binding. However, the AOS-like variants bound both the Notch activation complex and the Hairless/SHARP repression complexes with similar affinities as wild type $\mathrm{Su}(\mathrm{H}) / \mathrm{RBPJ}$. Altogether, these data suggest a sequestration mechanism, in which 
RBPJ/Su(H) AOS variants efficiently bind the Notch pathway co-activator and co-repressor proteins,

but their reduced DNA binding activity excludes these complexes from DNA and results in the misregulation of Notch target genes.

\section{RESULTS}

Identification of a Drosophila $\mathrm{Su}(\mathrm{H})$ allele with an analogous amino acid change as a human

\section{$R B P J$ variant associated with AOS}

Genetic studies in Drosophila have previously identified several $S u(H)$ alleles that were described as having dominant gain-of-function phenotypes ${ }^{15}$. To better understand the molecular function of these alleles, we sequenced the $S u(H)$ coding regions of the $S u(H)^{T 4}$ and $S u(H)^{O 5}$ alleles and found that both contain a single missense mutation within the same highly conserved domain (i.e. the N-terminal domain, or NTD) of $\mathrm{Su}(\mathrm{H})$, which is involved in DNA binding (Fig 1B). $\mathrm{Su}(\mathrm{H})^{\mathrm{O} 5}$ encodes a lysine to a methionine mutation $(\mathrm{K} 132 \mathrm{M})$, whereas $\mathrm{Su}(H)^{\mathrm{T4}}$ encodes a glutamic acid to valine mutation (E137V). Intriguingly, the E137 Su(H) residue corresponds to E63 in human RBPJ, which was found to be encoded by a missense variant (E63G) in a family with Adams Oliver Syndrome (AOS) ${ }^{14}$ (Fig 1C). However, unlike the gain-of-function activity ascribed to the fly $S u(H)^{T 4}$ allele, the RBPJ E63G variant was proposed to be a loss-of-function allele due to loss of DNA binding activity. Based on the crystal structures of RBPJ and Su(H) bound to DNA, both AOS variants identified by Hassed et. al. ${ }^{14}$ (E63G \& $\mathrm{K} 169 \mathrm{E})$ and both alleles identified by Fortini et. al. ${ }^{15}$ (K132M and E137V) are residues involved in specific and non-specific interactions with DNA (Fig 1D). It should be mentioned that while E63 and E137 correspond to the same glutamate residue in human and Drosophila RBPJ/Su(H), K169 and K132 are not homologous. Nonetheless, the discrepancy between the predicted gain-of-function activity of the Su(H) E137V variant in Drosophila and the predicted loss-of-function activity of the analogous human E63G variant provided an opportunity to reexamine the mechanisms underlying how the homologous $\mathrm{Su}(\mathrm{H})$ and RBPJ variants impact Notch signaling. 


\section{Su(H) and RBPJ AOS variants disrupt DNA binding}

To elucidate how the RBPJ/Su(H) variants affect function, we first performed comparative DNA binding assays using EMSAs with a probe containing a RBPJ/Su(H) binding site and purified recombinant proteins corresponding to Drosophila $\mathrm{Su}(\mathrm{H})$ and mouse Rbpj (Fig 2A, B, note we will refer to the mouse Rbpj protein with only the first letter capitalized, whereas human RBPJ is in all capital letters). We expressed and purified WT and AOS variants of mouse Rbpj (53-474) and Drosophila $\mathrm{Su}(\mathrm{H})(98-523)$, which corresponds to the highly conserved structural core of CSL proteins ${ }^{7}$. We used differential scanning fluorimetry to determine if the variants affect folding and overall stability of $\mathrm{Rbpj} / \mathrm{Su}(\mathrm{H})$. Consistent with these residues being surface exposed, none of the variants significantly affected folding/stability of $\mathrm{Rbpj} / \mathrm{Su}(\mathrm{H})$ compared to WT proteins except for mouse Rbpj E89G (analogous to human RBPJ E63G), which had a modest effect on its melting temperature $\left(\Delta \mathrm{T}_{\mathrm{m}}=-3.1^{\circ} \mathrm{C}\right.$, Supplemental Fig 1). EMSAs were performed with equimolar concentrations of WT $\mathrm{Su}(\mathrm{H})$ and the E137V [encoded by the $\mathrm{Su}(H)^{\mathrm{T} 4}$ allele] and $\mathrm{K} 132 \mathrm{M}$ [encoded by the $\mathrm{Su}(H)^{\mathrm{O} 5}$ allele] variants, which revealed that both variants decreased, but did not abolish, DNA binding to a high affinity site (Fig 2A). Quantitation of the EMSA data revealed that $\mathrm{Su}(\mathrm{H}) \mathrm{E} 137 \mathrm{~V}$ consistently had a stronger impact on DNA binding than the $\mathrm{Su}(\mathrm{H}) \mathrm{K} 132 \mathrm{M}$ variant. Similarly, purified Rbpj variants carrying analogous mouse amino acid variants (E89G and K195E) as those residues found in human associated AOS variants (E63G and K169E) also strongly weakened, but did not abolish DNA binding (Fig 2B). In this case, however, K195E had an even greater impact on DNA binding than E89G (Fig 2B). These results are further supported by EMSAs performed with the full-length mammalian proteins produced by cell free expression (Supplemental Fig 2).

Next, we used isothermal titration calorimetry (ITC) to quantitatively assess the changes in affinity and thermodynamics of these variant proteins binding to DNA (Fig 2C, D, Table S1). Each binding experiment was performed by titrating DNA containing a single $\mathrm{Rbpj} / \mathrm{Su}(\mathrm{H})$ binding site from a syringe into a calorimetric cell containing either purified $\mathrm{Su}(\mathrm{H})$ or RBPJ. WT $\mathrm{Su}(\mathrm{H})$ bound to DNA with a $\mathrm{K}_{d}$ of $188 \mathrm{nM}$ (Fig 2C, Table S1); whereas $\mathrm{Su}(\mathrm{H}) \mathrm{K} 132 \mathrm{M}$ bound to DNA with a $690 \mathrm{nM} \mathrm{K}$, showing 
an $~ 3.5$ fold reduction in binding, and $\mathrm{Su}(\mathrm{H}) \mathrm{E} 137 \mathrm{~V}$ bound to DNA with a $\mathrm{K}_{\mathrm{d}}$ of $842 \mathrm{nM}$ displaying an

-4.5 fold reduction in binding from WT (Fig 2C, Table S1). WT mouse Rbpj bound DNA with a 74 nM $\mathrm{K}_{\mathrm{d}}$, while the E89G and K169E variants had an 6- and 16-fold reduction in binding, respectively (Fig

2D, Table S1). These trends in reduced DNA binding are consistent with our EMSA data that similarly showed that the $\mathrm{Su}(\mathrm{H}) \mathrm{E} 137 \mathrm{~V}$ is more severe than $\mathrm{K} 132 \mathrm{M}$ and $\mathrm{Rbpj} \mathrm{K} 169 \mathrm{E}$ is more adversely affected than E89G (Fig 2A, B). From these experiments, we can conclude that the effects of these variants on DNA binding are conserved between the fly and human proteins, suggesting that the known Drosophila $S u(H)$ alleles can be used as a developmental model to study RBPJ variants that cause AOS in humans.

The $S u(H)$ AOS-like alleles exacerbate phenotypes associated with imbalanced Notch activator and Hairless co-repressor levels

Fortini and Artavanis-Tsakonas originally identified the $\mathrm{Su}(H)^{\mathrm{T} 4}$ and $\mathrm{Su}(H)^{\mathrm{O} 5}$ alleles in a genetic modifier screen of Notch pathway phenotypes ${ }^{15}$. In that study, they showed that these two Su(H) alleles altered Notch pathway phenotypes in sensitized backgrounds, and often in an opposing manner compared to $\mathrm{Su}(H)$ loss-of-function alleles. Hence, they classified these alleles as having "gain-offunction" activity. To better understand how each $S u(H)$ allele impacts Notch and Hairless phenotypes in light of our new sequencing results, we quantitatively reanalyzed two well established Notch $(N)$ sensitive phenotypes: wing nicking or "notches" that are found in Notch heterozygotes flies $\left(N^{+/-}\right)$; and macrochaetae sensory bristles, which increase in number with lower Notch levels (i.e. $N^{+/-}$) but decrease in number with lower Hairless $(H)$ co-repressor levels (i.e. $H^{+/-}$).

First, we directly compared the impact of having either a "gain-of-function" $\mathrm{Su}(H)^{\mathrm{T4}}$ or $\mathrm{Su}(H)^{\mathrm{O5}}$ allele or the "loss-of-function" $S u(H)^{\mid B 115}$ allele encoding a non-functional protein due to a premature stop codon (K138-stop) ${ }^{15,16}$. Flies with a single copy of the $S u(H)^{I B 115 /+}$ null allele in an otherwise WT genetic background developed normal wings (no nicks observed, N=174; Fig 3A). However, consistent with prior studies ${ }^{15}$, we found that a small subset of flies containing either a single copy of the $S u(H)^{T 4}$ 
or $\mathrm{Su}(H)^{\mathrm{O} 5}$ allele had a dominant wing nicking phenotype and quantitative analysis revealed that flies with the $S u(H)^{T 4}$ allele had both a higher penetrance and increased wing nicking severity than the Su(H) ${ }^{O 5}$ allele (Fig 3A, B). Thus, consistent with our quantitative in vitro DNA binding assays (see Fig 2), we found that the $S u(H)^{T 4}$ allele encoding the E137V variant has both a more dramatic impact on DNA binding and a stronger impact on wing nicking when compared to the $S u(H)^{\mathrm{O} 5}$ allele encoding the K132M variant.

To determine if the wing nicking phenotype in the more severe $S u(H)^{T 4}$ allele could be suppressed by compensatory changes in the Notch pathway, we first used GFP-tagged BAC transgenes to add an extra copy of either WT $S u(H)(S u(H)-G F P)$ or Notch (Notch-GFP) into flies carrying a single copy of the $S u(H)^{T 4}$ allele. Importantly, increasing the gene dose of both $S u(H)$ $\left(\mathrm{Su}(H)^{T 4} /+; \mathrm{Su}(H)-G F P\right)$ and Notch $\left(\mathrm{Su}(H)^{T 4} /+; N o t c h-G F P\right)$ suppressed the wing nicking phenotype (Fig 3A). Similarly, we found that removing a single copy of the Hairless gene that encodes a co-repressor that specifically antagonizes Notch pathway activation, also suppressed wing nicking in $S u(H)^{T 4 /+}$ flies $\left(S u(H)^{T 4 /+} ; H^{1 /+}\right)\left(\right.$ Fig 3A, C). Thus, the dominant wing phenotype caused by the $S u(H)^{T 4}$ allele can be suppressed by either increasing the WT dose of $S u(H)$, increasing the gene dose of Notch, or decreasing the gene dose of the $H$ co-repressor.

Next, we compared the ability of the $S u(H)^{T 4}, S u(H)^{O 5}$, and $S u(H)^{I B 115 /+}$ alleles to modify the wing nicking phenotype in Notch heterozygous animals that are known to have significant wing nicking in an animal with two WT $S u(H)$ alleles (Fig 3D, E). As expected, both the $S u(H)^{T 4}$ and $S u(H)^{O 5}$ alleles dramatically enhanced the wing notching phenotype in Notch heterozygous flies (i.e. $N^{55 e 11 /+} ; S u(H)^{T 4 /+}$ and $N^{55 e 11 /+} ; S u(H)^{05 /+}$ ) with the $S u(H)^{T 4}$ allele having a more dramatic impact on wing morphology (Fig 3D, F, G). In sharp contrast, the Notch haploinsufficiency wing phenotype was partially suppressed in flies with a single copy of the $S u(H)^{I B 115}$ null allele $\left(N^{55 e 11 /+} ; S u(H)^{I B 115 /+}\right)($ Fig 3D). Thus, while lowering the dose of $S u(H)$ using a null allele can partially alleviate the wing phenotype caused by too little Notch, the presence of the DNA binding compromised $S u(H)^{T 4}$ and $S u(H)^{O 5}$ alleles exacerbates this phenotype. 
The wing nicking phenotype in Notch heterozygous flies is thought to be caused by decreased Notch signal strength in the Cut-positive wing margin cells during larval development ${ }^{17-19}$. To better understand the impact of the $S u(H)^{T 4}$ and $S u(H)^{O 5}$ alleles on Notch signal strength within these cells, we performed comparative expression analysis of the $E(s p l) m a-G F P$ reporter gene in wing imaginal discs isolated from either WT, $S u(H)^{I B 1115 /+} S u(H)^{T 4} /+$ or $S u(H)^{05 /+}$ larva. $E(s p l) m \alpha$ is a known Notch target in the wing imaginal disc and is expressed in Cut+ wing margin cells as well as in additional cells that contribute to sensory organ development ${ }^{20,21}$ (Fig 4A). To assess Notch signal strength in wing margin cells, we performed quantitative GFP expression analysis using confocal microscopy (see Methods for details) and used a Cut-antibody to specifically label wing margin cells. Since E(spl)maGFP is activated very strongly in the macrochaetae lineage that develops along the anterior wing margin by proneural transcription factors, we focused our analysis on the easily isolated and visualized posterior Cut+ margin cells (labeled posterior in Fig 4A). Importantly, quantitative GFP analysis revealed that simply replacing a WT $S u(H)$ copy with the $S u(H)^{I B 115}$ null allele did not significantly impact GFP levels, whereas both the $S u(H)^{05}$ and $S u(H)^{T 4}$ alleles dramatically lowered GFP levels relative to WT and $S u(H)^{I B 115}$ heterozygotes (Fig 4B). These data are consistent with the AOS-like $S u(H)^{T 4}$ and $\mathrm{Su}(H)^{O 5}$ alleles, but not the $S u(H)^{I B 115}$ null allele, being sufficient to lower Notch signal strength in wing margin cells, and thereby induce wing nicks.

Prior studies of flies with a single copy of either the $S u(H)^{T 4}$ or $S u(H)^{O 5}$ allele also found that both enhance the loss of macrochaetae sensory organs in flies heterozygous for the Hairless $(H)$ corepressor gene ${ }^{15}$. In contrast, and as its name implies, a single copy of a $\mathrm{Su}(H)$ loss-of-function allele significantly suppresses the decrease in macrochaetae formation in Hairless heterozygous animals ${ }^{22,23}$. We similarly tested each of these $S u(H)$ alleles in flies with either two WT alleles of $H$ (i.e. a $y w^{1118}$ stock) or in flies with a single copy of Hairless $\left(H^{1 /+}\right)$. In the WT background, flies carrying a single copy of the $S u(H)^{I B 115}$ null allele invariably developed the expected 40 macrochaetae on the head and thorax of each adult fly (Fig 5A). Flies heterozygous for the $S u(H)^{O 5}$ and $S u(H)^{T 4}$ alleles also developed an average of 40 macrochaetae. However, unlike in $S u(H)^{I B 115}$ heterozygotes, we observed a small 
number of flies in both $\mathrm{Su}(H)^{\mathrm{O} 5}$ and $\mathrm{Su}(H)^{T 4}$ heterozygotes that either had 1 or 2 missing macrochaetae or had 1 extra macrochaetae (Fig $\mathbf{5 A}$ ). While the penetrance of these phenotypes is very low and the direction of change is variable (both increases and decreases in macrochaetae are observed), these data suggest that the process of macrochaetae selection and/or development may be less robust in $\mathrm{Su}(H)^{\mathrm{O5}}$ and $\mathrm{Su}(H)^{T 4}$ heterozygotes than in $\mathrm{Su}(H)^{I B 115}$ heterozygotes.

Next, we tested each allele in the $H^{1} /+$ heterozygotes that lose an average of $\sim 12$ macrochaetae (Fig 5B-F). As expected, replacing a WT $S u(H)$ allele with the $S u(H)^{I B 115}$ allele significantly suppressed

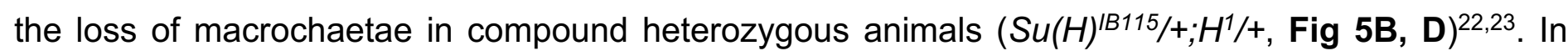
contrast, both the $S u(H)^{05}$ and $S u(H)^{T 4}$ alleles enhanced the loss of macrochaetae in $H^{1 / t}$ heterozygotes, and the $S u(H)^{T 4}$ allele again had a stronger impact than the $S u(H)^{O 5}$ allele (Fig $5 \mathbf{B}$, E, F). Next, we analyzed the impact of each of these $S u(H)$ alleles on macrochaetae phenotypes in flies sensitized to produce too many sensory bristles. Notch heterozygous flies produce a weakly penetrant, but significant increase in macrochaetae formation in animals with two WT alleles of Su(H) (Fig 5G). Intriguingly, we found that a single copy of either the $S u(H)^{T 4}$ or the $S u(H)^{O 5}$ allele further enhanced the number of extra macrochaetae in $N^{55 e 11 /+}$ flies (Fig 5G). In contrast, a single copy of the $S u(H)^{I B 115}$ null allele had the opposite effect and resulted in a small but significant suppression in the increase in macrochaetae in $N^{55 e 11 /+}$ flies.

Taken together, these data support two ideas: First, even within the same tissue, the presence of a single $\mathrm{Su}(H)^{T 4}$ or $\mathrm{Su}(H)^{\mathrm{O} 5}$ allele can significantly increase or decrease the number of sensory organs formed, but primarily only in sensitized genetic backgrounds that alter the amount of the Notch pathway co-activator (i.e. Notch) or co-repressor (i.e. Hairless) proteins. Second and in sharp contrast to the $S u(H)^{T 4}$ or $S u(H)^{O 5}$ alleles, we found that decreasing the gene dose of the WT $S u(H)$ allele using a single copy of a null allele (i.e. $\mathrm{Su}(H)^{\mid B 115 /+}$ ) partially suppressed each of these phenotypes in the same genetic backgrounds. These data suggest that simply lowering the amount of Su(H) transcription factor can suppress imbalances in Notch co-activator to Hairless co-repressor levels, whereas having a DNA binding compromised $\mathrm{Su}(\mathrm{H})$ molecule enhances these imbalances to make phenotypes worse. 
To further investigate how $\mathrm{Su}(H)$ gene dose impacts sensory organ formation, we generated a series of flies containing an additional copy of a WT $S u(H)$ allele (i.e. Su(H)-GFP BAC). In an otherwise WT background, flies containing a third copy of $\mathrm{Su}(\mathrm{H})$ were phenotypically normal and did not change the number of macrochaetae (Fig 5H). However, in Hairless heterozygotes, adding an extra copy of Su(H) (i.e. $\mathrm{Su}(H)^{+/+} ; H^{1} / \mathrm{Su}(H)-G F P$ ) enhanced the loss of macrochaetae (compare $H^{1 /+}$ data in Fig $\mathbf{5 G}$ with the $H^{1} / \mathrm{Su}(H)-G F P$ data in Fig $\left.\mathbf{5 H}\right)$, consistent with further increasing the levels of $\mathrm{Su}(\mathrm{H})$ transcription factor causing even stronger phenotypes. Next, we replaced one of the WT $S u(H)$ alleles with the $S u(H)^{T 4}$ allele (i.e. $S u(H)^{T 4 /+} ; H^{1} / S u(H)-G F P$ ) and found that these flies had an even larger loss in macrochaetae (Fig 5H). In contrast, replacing one of the WT $S u(H)$ alleles with the $S u(H)$ null allele (i.e. $\left.S u(H)^{I B 115 /+} ; H^{1} / S u(H)-G F P\right)$ resulted in flies with a similar number of macrochaetae as $H^{1 /+}$ heterozygotes (Fig 5G, H). Altogether, these data suggest that the amount of Su(H) transcription factor is critical for proper Notch signaling and if one of these alleles encodes a $\mathrm{Su}(\mathrm{H})$ transcription factor with compromised DNA binding activity the phenotypes are dramatically enhanced, especially in flies with imbalanced levels of Notch and Hairless.

\section{Su(H) and RBPJ AOS variants bind normally to the NICD co-activator and the Hairless/SHARP}

\section{co-repressor proteins}

Integrating the DNA binding and genetic data suggests the following model: the $S u(H)^{T 4}$ and $\mathrm{Su}(H)^{\mathrm{O} 5}$ alleles produce proteins that sequester the NICD co-activator and Hairless co-repressor off DNA. Hence, the presence of a single copy of either $S u(H)^{\top 4}$ or $S u(H)^{O 5}$ can exacerbate both wing notching and the gain in macrochaetae when Notch is limiting $\left(N^{55 e 11 /+}\right)$, and the loss in macrochaetae when Hairless is limiting $\left(H^{1 /+}\right)$. To further test this idea, we performed ITC assays to directly measure $\mathrm{Su}(\mathrm{H})$ and RBPJ binding to both co-activators and co-repressors. We hypothesized that the DNA binding mutations in $\mathrm{Su}(\mathrm{H}) / \mathrm{RBPJ}$ would not impact cofactor binding because these sites are distant from the interfaces of Su(H)/RBPJ involved in co-activator and co-repressor binding (Fig 6A). To test the $\mathrm{Su}(\mathrm{H})$ variants' ability to bind co-activators, we performed ITC with a construct that corresponds to 
the RAM domain of the Drosophila Notch receptor, which binds the $\beta$-trefoil domain (BTD) of $\mathrm{Su}(\mathrm{H})$ with high affinity ${ }^{24}$ (Fig 6A). WT Su(H) bound to RAM with $187 \mathrm{nM}$ affinity, whereas we observed no statistically significant differences in RAM binding to Su(H) E137V [T4] or K132M [O5] (Fig 6B, Table S1). To test the Su(H) variants' ability to bind co-repressors, we performed ITC with Hairless, residues 232-358, which correspond to the region of Hairless that binds the C-terminal domain (CTD) of $\mathrm{Su}(\mathrm{H})$ with high affinity ${ }^{25,26}(\mathrm{Fig}$ 6A). WT Su(H) bound Hairless with a $3 \mathrm{nM} \mathrm{K}$ and we observed no significant differences in Hairless binding to Su(H) E137V or K132M (Fig 6C, Table S1).

To test if mouse Rbpj proteins with the AOS variants showed similar trends with respect to coactivator/repressor binding, we performed ITC with the mouse RAM domain from Notch1 and the mouse co-repressor SHARP ${ }^{27-29}$. WT Rbpj and the E89G and K195E variants similarly bound RAM with an $\sim 20 \mathrm{nM} \mathrm{K}_{\mathrm{d}}$ (Fig 6D, Table S1). For Rbpj-SHARP complexes, WT and E89G bound the mouse SHARP protein with $\sim 5 \mathrm{nM}$ affinity, whereas $\mathrm{K} 169 \mathrm{E}$ bound with a $21 \mathrm{nM} \mathrm{K}$; however, this difference was not found to be statistically significant (T-test $p$ value $=0.079)$. To further confirm that coactivator/repressor binding was unaffected for the mouse Rpbj protein containing analogous AOS variants, we performed coimmunoprecipitation experiments in HEK293 cells transfected with epitopetagged versions (either GFP- or FLAG-tagged as indicated) of each Rbpj variant in the presence of either NICD1, NICD1 + MAML1, or SHARP (Fig 6F-H). NICD1 coimmunoprecipitated with each mouse Rbpj AOS variants to a similar extent as WT Rbpj (Fig 6F) and MAML1 coimmunoprecipitated with NICD1 and Rbpj to similar levels with WT and variant Rbpj constructs (Fig 6G). Both mouse Rbpj AOS variants also coimmunoprecipitated equally well with SHARP compared to WT Rbpj (Fig 6H). Taken together, these data support the hypothesis that the $\mathrm{Su}(\mathrm{H})$ variants and mouse Rbpj AOS variants specifically disrupt DNA binding without affecting co-activator/repressor binding, corroborating a model of cofactor sequestration. 
To confirm that the mouse Rbpj AOS variants do not impact protein subcellular localization, we transfected HeLa cells with GFP tagged Rbpj and monitored protein distribution using fluorescence microscopy. Like WT Rbpj, both AOS variants were primarily localized in the nucleus (Fig 7A). Additionally, we treated Rbpj transfected cells with cycloheximide and monitored protein half-life. The degradation rates of both Rbpj AOS variants were similar to WT Rbpj (Supplemental Fig 3). We performed luciferase assays using a reporter containing 12 CSL binding sites in HeLa ${ }^{\text {RBPJ-KO }}$ cells, in which endogenous RBPJ had been depleted using CRISPR-Cas9 ${ }^{30}$, to test whether the AOS variants could bind and activate the reporter in the presence and absence of NICD1 (Fig 7B, C). First, we either expressed a fusion protein of wild type mouse Rbpj or the mouse Rbpj protein with AOS-variants to the VP16 activation domain to determine whether the AOS variants could activate the reporter in a NICD1 independent manner. Both Rbpj K195E-VP16 and E89G-VP16 fusions showed significant reduction in reporter signal compared to WT Rbpj-VP16, consistent with the above DNA binding experiments that show each variant protein has decreased DNA binding activity (Fig 7B). To investigate the effect of these AOS variants on Notch-mediated transcription, we performed luciferase assays by cotransfection with mouse NICD1 and Rbpj. In this case, we observed robust signaling with WT Rbpj and NICD1, whereas Rbpj K195E and E89G activated the reporter to a much lesser extent than WT. Moreover, it should be noted that the K195E variant showed a stronger impact on transcriptional responses when either fused to VP16 or co-transfected with NICD1 than the E89G variant, consistent with the K195E variant have a more dramatic impact on DNA binding.

Lastly, we assessed the ability of each Rbpj variant to repress target genes using a mature $\mathrm{T}$ (MT) cell line in which Notch signaling is in the "off" state. In this system, CRISPR/Cas9-mediated depletion of Rbpj leads to upregulation of Notch target genes, due to de-repression, and this phenotype can be efficiently rescued by reintroducing WT mouse Rbpj expression via lentiviral transduction ${ }^{27}$. We generated WT Rbpj, E89G, and K195E MT cell lines and performed qRT-PCR to quantify each protein's ability to repress transcription at the Hey1 and Hes1 Notch target sites (Fig 7D). As expected, expression of WT Rbpj rescued repression of Hey1 and Hes1, whereas the Rbpj K195E protein that 
more strongly affects DNA binding (Fig 2), failed to repress transcription at both Hey1 and Hes1 sites.

However, Rbpj E89G, which has a lesser impact on DNA binding than K195E (Fig 2), restores repression at Hey1 and Hes1 similar to WT RBPJ. Taken together, these results suggest that the severity of the mutation on DNA binding directly relates to the level of both transcriptional activation and repression.

\section{DISCUSSION}

Human genetic studies previously revealed that Adams Oliver Syndrome (AOS) is associated with autosomal dominant alleles in the Notch1, DIl4, and RBPJ genes ${ }^{31}$. Sequence analysis of these Notch pathway variants has led to the prediction that each variant is likely to compromise Notch signaling in specific tissues, and thereby cause a developmental syndrome that affects multiple organ systems. In this study, we took advantage of the unexpected finding that a previously described dominant gain-of-function $\mathrm{Su}(H)$ allele in Drosophila ${ }^{15}$ contains a missense variant in an analogous residue found in a family with $\mathrm{AOS}^{14}$. By combining quantitative genetic studies with quantitative DNA binding, protein-protein interaction, and transcriptional reporter assays, our findings provide evidence that $\mathrm{Su}(\mathrm{H}) / \mathrm{Rbpj}$ variants that compromise DNA binding can result in Notch target gene misregulation and phenotypes due to the sequestration of either the Notch signal or the antagonistic co-repressor proteins. Moreover, our findings reveal how increasing or decreasing the genetic dose of $\mathrm{Su}(H)$ can either suppress or exacerbate Notch pathway phenotypes depending on genetic background. Taken together, these findings have several important implications for better understanding the potential molecular defects underlying both AOS phenotypes and Notch pathway dysregulation.

First, our studies in Drosophila highlight that $S u(H)$ allelic variants that specifically encode proteins with altered DNA binding, but not co-activator/co-repressor binding, behave very differently than null $S u(H)$ alleles that fail to generate any functional protein. In particular, we found that the presence of a single $\mathrm{Su}(H)$ allele encoding a DNA compromised protein strongly enhances Notch phenotypes, whereas simply lowering the genetic dose of $\mathrm{Su}(H)$ using a protein null allele partially 
suppresses Notch phenotypes. Interestingly, since the discovery of the AOS-associated E63G and K169E human RBPJ variants that were originally identified in $2012^{14}$, additional RBPJ AOS variants have been identified, including R65G, F66V, and S332R ${ }^{31}$. Importantly, none of the AOS-associated RBPJ alleles are predicted to be a protein null allele and given the role of R65 and F66 in DNA binding, as shown in previous Rbpj structures ${ }^{29}$, and their close proximity to E63 in the NTD of RBPJ, these missense variants will also likely reduce DNA binding, while having little to no effect on cofactor binding. S332, which is currently classified as a variant of unknown significance ${ }^{32}$, does not directly contact DNA, but rather is located in a long $\beta$-strand that connects the BTD and CTD of RBPJ. However, it is possible that the S332R AOS variant will incur structural changes that indirectly decrease the overall binding affinity for DNA, resulting in a similar disease mechanism. Nonetheless, we have established a rigorous platform to test these and future AOS variants in vitro and in vivo.

Intriguingly, an analogous cellular mechanism that compromises $\mathrm{Su}(\mathrm{H})$ DNA binding has previously been identified in Drosophila ${ }^{33,34}$. In this case, phosphorylation of Su(H) residue S269, which interacts with DNA, blocks DNA binding, and plays a role in fly hematopoiesis. Moreover, it is tempting to speculate that the severity of AOS disease is potentially linked to the impact the variant has on DNA binding. Certainly, our genetic studies in Drosophila support this premise, i.e. the $S u(H)^{T 4}$ allele encoding the E137V variant had a stronger impact on DNA binding in our in vitro assays than the $\mathrm{Su}(H)^{\mathrm{O} 5}$ allele encoding the $\mathrm{K} 132 \mathrm{M}$ variant (Fig 2), and the $\mathrm{Su}(H)^{\mathrm{T} 4}$ allele consistently led to stronger mutant phenotypes in vivo (Fig 3-5). Perhaps some of the patient variability can be explained by the severity of the RBPJ variant; however, there are likely other genetic and environmental modifiers that also contribute to an individual's disease presentation, obscuring the relative effect a variant has on DNA binding.

Second, our studies, as well as the original publication that identified the $S u(H)^{T 4}$ and $S u(H)^{05}$ alleles $^{15}$, revealed strong genetic interactions with not only Notch, but also the antagonistic Hairless corepressor gene. These findings raise the possibility that human AOS-associated RBPJ alleles with compromised DNA binding may cause developmental defects due to the sequestration of mammalian 
co-repressor proteins and not simply due to sequestration of the Notch signal. However, it should be noted that repression mechanisms are not as well conserved across species, as there is no direct Hairless ortholog in mammals and mammalian RBPJ can bind multiple different co-repressors $^{10}$. Moreover, we found that only the stronger AOS variant Rbpj K195E was defective in the MT cell repression assay (Fig 7D), whereas Rbpj E89G, which has a milder defect in DNA binding, functioned similarly to WT in this repression assay. Additionally, no known variants in Notch pathway co-repressor genes have been associated with AOS, whereas numerous NOTCH1 and DLL4 variants have been associated with this developmental syndrome ${ }^{31}$, and several of the autosomal dominant NOTCH1 and DLL4 AOS alleles encode nonsense variants that are unlikely to generate a protein with dominantnegative effects. Thus, the clinical similarities between AOS patients with variants in NOTCH1, DLL4, and RBPJ suggest that the majority of developmental defects associated with RBPJ variants that encode proteins with compromised DNA binding are likely due to decreased NOTCH1 signal strength and not due to an impact on the antagonistic co-repressors. Nevertheless, since the genetic etiology of human patients with AOS is far from complete, future studies may want to include sequence analysis for variants in co-repressor genes known to interact with RBPJ.

Third, our studies and proposed sequestration disease mechanism raise an interesting unanswered question - since RBPJ is the sole downstream nuclear effector of Notch signaling, why do AOS patients harboring RBPJ variants have distinct and nonoverlapping phenotypes with Alagille syndrome patients that have variants in $\mathrm{NOTCH} 2$ or $\mathrm{JAG} 1^{13}$ ? Current data suggests that the $\mathrm{NOTCH}$ signal (i.e. the intracellular domains) generated by both NOTCH1 and NOTCH2 interact with RBPJ with similar affinities ${ }^{35,36}$, suggesting that the DNA binding compromised RBPJ proteins should similarly sequester the signals generated by either $\mathrm{NOTCH} 1$ or NOTCH2. Hence, it is unclear why the AOSassociated RBPJ alleles that cause similar phenotypes as the AOS-associated NOTCH1 variants do not also cause Alagille-like phenotypes that have been associated with NOTCH2 variants that cause decreased $\mathrm{NOTCH} 2$ activity. While answering this question is beyond the scope of this study, 
developing vertebrate models of AOS will certainly help address this question and provide further insights into the molecular mechanism of AOS, Alagille, and Notch signaling in general.

\section{Acknowledgements}

We thank the Drosophila stock centers and Developmental Studies Hybridoma Bank (DSHB) for fly stocks and antibody reagents. We also thank Sabine Schirmer and Roswitha Rittelmann for excellent technical assistance.

Declaration of interests. R.A.K is on the scientific advisory board of Cellestia Biotech AG and has received research funding from Cellestia for projects unrelated to this manuscript. The remaining authors declare no competing interests.

Funding. This work was supported by NSF/MCB-BSF grant \#1715822 and \#2114950 (B.G. and R.A.K.) and NIH grant CA178974 (R.A.K.); the Deutsche Forschungsgemeinschaft (German Research Foundation; SFB 1074/A03, OS 287/4-1 and GRK 2254/C4) and the "Deutsche Krebshilfe" (German Cancer Aid, \#70114289) to F.O.; the Deutsche Forschungsgemeinschaft (DFG, German Research Foundation) - Project number 109546710 - TRR81 and BO 1639/9-1 and the Von-Behring-Röntgen foundation and the Excellence Cluster for Cardio Pulmonary System (ECCPS) to T.B.;

Contributions: This scientific study was conceived and planned by E.K.G., L.M.G., Y.K., R.A.K., and B.G. Additional experiments were planned by F.O., T.B., and P.J.D. The $\mathrm{Su}(\mathrm{H})$ alleles were originally sequenced by P.J.D. and E.H.T. and independently confirmed by E.K.G. The Drosophila genetic experiments and quantitative imaging experiments were performed by L.M.G. All bacterial protein purifications and ITC experiments were performed by E.K.G. The EMSA experiments in Figure 2 were performed by Y.K. and S.H. performed EMSA experiments in Figure S2. P.H. performed IPS, luciferase 
assays, and MTHs in Figures 6-7 and A.T. performed the repression assay in Figure 7. The manuscript was written by E.K.G., R.A.K., and B.G. and edited by all authors.

\section{Figure Legends}

Figure 1. Variants in the Notch pathway transcription factor associated with Adams Oliver

\section{Syndrome}

A. Overview of the Notch signaling pathway. Signal sending cells that express Notch ligands (DSL) interact with adjacent signal receiving cells that express Notch receptors. Upon receptor-ligand binding, the Notch receptor is cleaved by $\mathrm{Y}$-secretase, resulting in the release of the soluble Notch Intracellular Domain (NICD). NICD binds to the CSL transcription factor and recruits the co-activator Mastermind (MAM) to activate transcription of Notch target genes. The CSL transcription factor can also engage co-repressors (CoR) to inhibit transcription.

B. Sequence traces from PCR amplified genomic DNA isolated from flies heterozygous for the $S u(H)^{\top 4}$ and $\mathrm{Su}(H)^{\mathrm{O} 5}$ alleles reveal missense mutations (arrows) in each respective fly line.

C. Top: Linear domain layout of CSL. NTD = N-terminal domain, BTD = beta-trefoil domain, CTD = Cterminal domain. Grey regions are disordered and poorly conserved between species. Bottom: Multiple sequence alignments of a portion of the human, mouse, and Drosophila NTD of CSL with residues that are $100 \%$ conserved highlighted in cyan. Yellow highlighting indicates the residues altered due to missense variants in two separate families with AOS (specific amino acid changes listed in red text) and the missense variants found in the $S u(H)$ alleles (specific amino acid changes listed in green text). D. Middle: Ribbon diagram of RBPJ bound to DNA (PDBID 3IAG) ${ }^{37}$, with the same domain coloring as described in C and the DNA shown in purple. Left: Close up view of the K169/195/243 residue in yellow, numbered according to human (red), mouse (blue), and Drosophila (green) respectively. Right: Close up view of the $E 63 / 89 / 137$ and $\mathrm{K} 58 / 84 / 132$ residues in yellow, numbered according to the human (red), mouse (blue), and Drosophila proteins respectively. Black dashed lines indicate hydrogen bonding. 


\section{Figure 2. $\mathrm{Su}(\mathrm{H})$ and RBPJ AOS variants decrease DNA binding.}

A-B. Representative EMSAs with triplicate quantification shown on the right for purified (A) Drosophila Su(H) WT, E137V, and K132M proteins and (B) mouse Rbpj WT, E89G, and K195E proteins. P-values are reported for the $125 \mathrm{nM}$ protein conditions and were determined by an ANOVA with Tukey Honest Significant Difference test $\left(\left[{ }^{\star \star *}\right] \mathrm{P}<0.001,\left[{ }^{\star *}\right] \mathrm{P}<0.01,\left[{ }^{\star}\right] \mathrm{P}<0.05\right.$ and $[\mathrm{NS}]$ not significant). WT Su(H) vs. $\mathrm{Su}(\mathrm{H}) \mathrm{K} 132 \mathrm{M} \mathrm{P}=2.14 \mathrm{E}-6$. WT $\mathrm{Su}(\mathrm{H})$ vs. E137V P=9.9E-9. WT RBPJ vs. E89G P=2.27E-8. WT RBPJ vs. K195E P=6.42E-14.

C-D. Representative thermograms showing the raw heat signal and nonlinear least squares fit to the integrated data for $(\mathrm{C})$ Drosophila $\mathrm{Su}(\mathrm{H})$ and $(\mathrm{D})$ mouse Rbpj variants binding to a 20 mer oligo duplex containing the same RBPJ/Su(H) binding site used in the above EMSAs. Each ITC experiment was performed at $10^{\circ} \mathrm{C}$ with 20 injections. The mean dissociation constants $\left(K_{D}\right)$ and standard deviations from triplicate experiments are reported along with the p-value determined from a two-tailed T-test $\left(\left[{ }^{\star \star \star}\right]\right.$ $\left.\left.\mathrm{P}<0.001,{ }^{* \star}\right] \mathrm{P}<0.01,{ }^{\star}\right] \mathrm{P}<0.05$ and $[N S]$ not significant). WT Su(H) vs. Su(H) K132M P=2.22E-4. WT Su(H) vs. E137V P=8.22E-4. WT RBPJ vs. E89G P=4.06E-3. WT RBPJ vs. K195E P=3.97E-3.

Figure 3. AOS-like $S u(H)$ alleles induce wing nicking phenotypes that can be enhanced or suppressed by genetic changes in the Notch signaling pathway.

A. Quantified wing nicking in the indicated genotypes with number of wings analyzed $(n)$ noted on each bar graph. Flies used in the top three bars were generated by crossing the $y w^{1118}$ stock that is WT for $\mathrm{Su}(H)$ with flies carrying each of the indicated $\mathrm{Su}(H)$ alleles. Flies used in the bottom three bars were generated by crossing flies carrying a $\mathrm{Su}(H)^{T 4}$ allele with flies carrying either the $S u(H)$-GFP BAC, the Notch-GFP BAC, or the $H^{1}$ null allele, as indicated. Proportional odds model with Bonferroni adjustment tested for penetrance/severity differences between $S u(H)^{O 5 /+}$ and $S u(H)^{T 4 /+}$ flies with $\bullet$ denoting $p<0.01$. Two-sided Fisher's exact test was used to assess all other genotypes with * denoting $p<0.01$.

B-C. A wing from a Su(H $)^{T 4 /+}$ heterozygote in an otherwise WT background (B) or in combination with a $H^{1 /+}$ heterozygous background $\left(\mathrm{Su}(H)^{T 4 /+} ; H^{1 /+}, \mathrm{C}\right)$. Note, the $\mathrm{Su}(H)^{T 4 /+}$ wing had a nick (black 
arrowhead), whereas all the wings from $S u(H)^{T 4 /+} ; H^{1 /+}$ flies did not have wing nicks.

D. Quantified wing notching in the indicated genotypes with number of wings analyzed ( $\mathrm{n})$ noted on each bar graph. Flies were generated by crossing $N^{55 e 11 /+}$ female flies with either $y w^{1118}$ WT male flies or male flies carrying the indicated $S u(H)$ alleles. Proportional odds model with Bonferroni adjustment tested for penetrance/severity differences between $S u(H)^{05 /+}$ and $S u(H)^{T 4 /+}$ flies with $\bullet$ denoting $p<0.01$

E-G. Wings from female flies containing a copy of the $N^{55 e 11}$ allele in either a WT $(E), S u(H)^{T 4 /+}(F)$ or $\mathrm{Su}(H)^{\mathrm{O} /+}(\mathrm{G})$ background. Note, the severe nicking and morphological wing phenotypes observed in the compound heterozygotes.

Figure 4. Flies with an AOS-like $S u(H)$ allele have dramatically lower expression of a Notch reporter gene in wing margin cells.

A. Representative expression of the E(spl)ma-GFP reporter in larval wing discs from WT flies (far left) or from flies heterozygous for either $S u(H)^{I B 115}, S u(H)^{O 5}$, or $S u(H)^{T 4}$, as indicated at top. Note, the wing discs were immunostained for Cut (red) to mark the wing margin cells and only the posterior margin cells were quantified in B. Bottom panel shows GFP expression in grayscale.

B. Quantification of GFP levels from wing discs of the indicated genotypes. Each dot represents the average posterior wing margin cell pixel intensity as measured from an individual imaginal disc. * denotes significance $(p<0.01)$ using a two-sided Student's t-test.

Figure 5. AOS-like $\mathrm{Su}(\mathrm{H})$ alleles can enhance both macrochaetae loss or gain depending upon Notch and Hairless gene dose.

A. Quantified macrochaetae numbers in flies carrying either a single copy of $S u(H)^{I B 115}, S u(H)^{O 5}$, or $\mathrm{Su}(H)^{T 4}$, as indicated. Number of animals analyzed $(\mathrm{n})$ noted on each bar graph. Note, flies heterozygous for the $S u(H)^{I B 115}$ allele produce the expected invariable 40 macrochaetae on the head/thorax and show no variability. In contrast, a small percentage of both the $S u(H)^{05}$ and $S u(H)^{T 4}$ 
heterozygous flies either lost or gained a small number of macrochaetae. Significance was calculated using a two-sided Fisher's exact test.

B. Quantified macrochaetae numbers in flies of the following genotypes: WT $(+/+)$; Hairless heterozygotes $\left(H^{1 /+}\right)$; and $S u(H) / H^{1}$ compound heterozygotes with either the $S u(H)^{I B 115}, S u(H)^{O 5}$, or $S u(H)^{T 4}$ alleles as indicated. Number of animals analyzed $(\mathrm{n})$ noted below each genotype. Note, flies heterozygous for the $S u(H)^{I B 115}$ allele suppress the $H^{1}$ loss of macrochaetae, whereas both $S u(H)^{05}$ and $\mathrm{Su}(H)^{T 4}$ compound heterozygous flies enhance macrochaetae loss in a Hairless heterozygous background. Significance was calculated using a two-sided Student's t-test.

C-F. Image of a Drosophila notum and head showing the loss of macrochaetae (marked by blue asterisks) in flies with the following genotypes: $H^{1 /+}(\mathrm{C}), S u(H)^{1 B 115 /+} ; H^{1 /+}(\mathrm{D}), S u(H)^{T 4 /+} ; H^{1 /+}(\mathrm{E}) ;$ or $\mathrm{Su}(H)^{\mathrm{O} /++} ; H^{1 /+}(\mathrm{F})$

G. Quantified macrochaetae numbers in flies of the following genotypes: WT (+/+); Notch heterozygotes $\left(N^{55 e 11 /+}\right)$; and $N^{55 e 11} / S u(H)$ compound heterozygotes with either the $S u(H)^{I B 115}, S u(H)^{O 5}$, or $S u(H)^{T 4}$ alleles as indicated. Number of animals analyzed ( $n$ ) noted below each genotype. Note, flies heterozygous for the $S u(H)^{I B 115}$ allele suppressed the $N^{55 e 11}$ gain of macrochaetae, whereas both the $\mathrm{Su}(H)^{\mathrm{O5}}$ and $\mathrm{Su}(H)^{T 4}$ heterozygous flies enhanced macrochaetae gain in a Notch heterozygous background. Significance was calculated using a two-sided Student's t-test.

H. Quantified macrochaetae numbers in WT flies (+/+), flies carrying an extra copy of $S u(H)(S u(H)-$ GFP), flies with an extra copy of $S u(H)$ and heterozygous for Hairless $\left(H^{1} / S u(H)-G F P\right)$, flies with $\operatorname{Su}(H)^{T 4 /+} ; H^{1} / S u(H)-G F P$, flies with $S u(H)^{I B 115} /+; H^{1} / S u(H)-G F P$, and flies with $S u(H)^{I B 115 /+; H^{1 /+}}$ as indicated. Number of animals analyzed ( $n$ ) noted below each genotype. Significance was calculated using a two-sided Student's t-test.

Figure 6. The Su(H)/RBPJ variants bind the co-activator and co-repressor proteins as well as WT Su(H)/RBPJ. 
A. Structures of the RAM domain (left, PDBID: 3V79) ${ }^{38}$, Hairless (middle, PDBID: 5E24) ${ }^{25}$, and SHARP

(right, PDBID: 6DKS) ${ }^{27}$ bound to $\mathrm{Su}(\mathrm{H}) / \mathrm{RBPJ}$, with DNA removed from the model for simplicity. $\mathrm{Su}(\mathrm{H}) / \mathrm{RBPJ}$ is represented as a surface colored by domains as in Figure $1 \mathrm{C}$, with the $\mathrm{Su}(\mathrm{H})$ and $\mathrm{RBPJ}$ DNA binding variants of the NTD colored yellow and labeled DBM (DNA Binding Mutation). The cofactors are represented as solid cartoons with RAM in red, Hairless in purple, and SHARP in pink.

B-E. Representative thermograms showing the raw heat signal and nonlinear least squares fit to the integrated data from ITC experiments with (B) Drosophila Notch RAM in the syringe and $\mathrm{Su}(\mathrm{H})$ in the cell, (C) Drosophila Hairless in the syringe and $\mathrm{Su}(\mathrm{H})$ in the cell, (D) mouse Notch 1 RAM in the syringe and mouse Rbpj in the cell, and (E) mouse SHARP in the syringe and mouse Rbpj in the cell. Each ITC experiment was performed at $25^{\circ} \mathrm{C}$ with 20 injections. The average dissociation constants $\left(K_{D}\right)$ and standard deviations from triplicate experiments are reported along with the p-value determined from a two-tailed T-test $\left(\left[{ }^{* * *}\right] \mathrm{P}<0.001,\left[{ }^{* *}\right] \mathrm{P}<0.01,\left[{ }^{*}\right] \mathrm{P}<0.05\right.$ and $[\mathrm{NS}]$ not significant). For the RAM binding experiments: WT Su(H) vs. Su(H) K132M P=0.142. WT Su(H) vs. E137V P=0.583. WT RBPJ vs. E89G $P=0.860$. WT RBPJ vs. K195E $P=0.789$. For the Hairless/SHARP binding experiments: WT $\mathrm{Su}(\mathrm{H})$ vs. $\mathrm{Su}(\mathrm{H}) \mathrm{K} 132 \mathrm{M} P=0.605$. WT Su(H) vs. E137V $P=0.185$. WT RBPJ vs. E89G $P=0.614$. WT RBPJ vs. K195E $P=0.079$.

F. NICD binding of WT and variant mouse Rbpj proteins from cells. HEK293 cells were transfected with the plasmids GFP-Rbpj WT, GFP-Rbpj E89G, or GFP-Rbpj K195E in the absence or presence of FlagNICD. Immunoprecipitation was performed with anti-Flag antibody agarose beads and detected by Western blotting using an anti-GFP antibody. Expression of GFP-Rbpj (middle blot) was detected using an anti-GFP antibody. Expression of the Flag-NICD protein (bottom blot) was detected using an antiFlag antibody. The asterisk in the upper blot marks the heavy chain of the antibody used for immunoprecipitation.

G. Mouse Rbpj WT and Rbpj-AOS variants similarly form the NICD co-activator complex that includes Mam1. HEK293 cells were transfected with the plasmids expressing Maml1 in the absence or presence of Flag-Rbpj WT, Flag-Rbpj E89G, or Flag-Rbpj K195E and Flag-NICD. Co-immunoprecipitation was 
performed with the anti-Flag antibody agarose beads and detected by Western blotting using an anti-

Maml1 antibody. Expression of Flag-Rbpj proteins (middle blot) and Flag-NICD was detected using an anti-Flag antibody. Expression of the Maml1 protein (bottom blot) was detected using the anti-Maml1 antibody.

H. CoIP of mouse SHARP with WT and AOS variants of Rbpj. HEK293 cells were transfected with the plasmids GFP-Rbpj WT, GFP-Rbpj E89G, or GFP-Rbpj K195E in the absence or presence of FlagSHARP-(RBPID, Rbpj-interaction domain). Immunoprecipitation was performed with the anti-Flag antibody agarose beads and detected by Western blotting using an anti-GFP antibody. Expression of GFP-Rbpj (middle blot) was detected using an anti-GFP antibody. Expression of the Flag-SHARP(RBPID) protein (bottom blot) was detected using an anti-Flag antibody. The asterisk in the upper blot marks the heavy chain of the antibody used for immunoprecipitation.

\section{Figure 7: The RBPJ AOS variants show dysregulated Notch target gene expression when compared to WT RBPJ.}

A. Subcellular localization of Rbpj proteins after expression in HeLa cells, illustrating predominantly nuclear localization of both WT and variant Rbpj proteins. HeLa cells were transiently transfected with $0.3 \mu \mathrm{g}$ of the respective GFP-Rbpj plasmids: WT, E89G, and K195E. After 24 hours, cells were fixed and stained with DAPI and imaged under a fluorescent microscope using a $63 x$ objective.

B. Luciferase reporter assay using an Rbpj-VP16 fusion to test for DNA binding capacities (schematic lower panel). Both Rbpj K195E-VP16 and E89G-VP16 show reduced reporter signal compared to WT, consistent with the observation that both have impaired DNA binding. HeLaRBPJ-KO cells were transfected with the Notch/RBPJ dependent reporter 12xCSL-RE-Luc (250 ng) without or with the indicated Rbpj-VP16 constructs (10 ng). Luciferase activity was measured 24 hours after transfection. Bars represent mean values from six independent experiments; error bars indicate standard deviation, ${ }^{* * *} p<0.001$. 
C. Transactivation capacities of RBPJ WT and AOS variants together with NICD. HeLaRBPJ-KO cells were transfected with the Notch/RBPJ dependent reporter 12xCSL-RE-Luc (250 ng) without or with the indicated Rbpj constructs (50 ng) and the NICD expression plasmid (10 ng) (schematic lower panel). In RBPJ depleted HeLa cells, NICD is not able to transactivate the reporter. Together with NICD, the AOS Rbpj variants show decreased transactivation capacities compared to Rbpj WT. Luciferase activity was measured 24 hours after transfection. Bars represent mean values from six independent experiments; error bars indicate standard deviation, ${ }^{* * *} p<0.001$.

D. Rbpj-WT but not the AOS variant K195E are able to rescue transcription repression of Notch target genes. Left: Western blot against Rbpj (WT and variants) in reconstitution experiments using CRISPR/Cas9-mediated depletion of Rbpj in mature T (MT) cells. Rbpj protein expression levels were analyzed in Rbpj-depleted cells (control, line 1), Rbpj WT or Rbpj E89G and Rbpj K195E mutants, (lanes 2,3 and 4 respectively) and TBP was used as a loading control. Right: qRT-PCR: Expression of Rbpj WT and Rbpj E89G, but not Rbpj K195E rescue the repression of Hey1 and Hes1 Notch target genes. Total RNA was extracted from Rbpj depleted mature T cells reconstituted with either empty vector (control), Rbpj WT and Rbpj E89G or Rbpj K195E mutants. Data shown were normalized to the housekeeping gene Bact and represent the mean \pm SD of three independent experiments $\left(\left[{ }^{* * *}\right] P<\right.$ 0.001, $\left.\left.{ }^{* *}\right] \mathrm{P}<0.01,{ }^{*}\right] \mathrm{P}<0.05$ and $[\mathrm{NS}]$ not significant, unpaired Student's t-test). 


\section{Methods}

Bacterial expression constructs: $\mathrm{Su}(\mathrm{H})$ amino acid residues $98-523$ corresponding to the conserved and structurally ordered core domain were cloned into the pGEX-6P1 bacterial expression vector ${ }^{24}$. Based on previous studies ${ }^{26}$, additional mutations R155T and N218G, which are surface exposed and distal from sites of DNA and co-regulator binding, were introduced to improve upon protein purification, yield, and stability. These mutations were previously shown to have identical binding to Notch, Hairless, and DNA as WT Su(H) ${ }^{26}$. Quick-change site directed mutagenesis was used to introduce the mutations $\mathrm{K} 132 \mathrm{M}$ and E137V into the pGEX-6P1-Su(H) construct. For the mammalian ortholog, mouse Rbpj amino acids 53-474, corresponding to the structural core, were cloned into pGEX-6P1 and the E89G and K195E variants were introduced as mentioned above ${ }^{29}$.

Mammalian expression constructs: The expression plasmids pcDNA3-Flag-hsNotch-1-IC (Flag-NICD), pcDNA3-Flag-Rbpj(WT), pcDNA3-Rbpj-VP16(WT), pcDNA-3.1-Flag1-hsSHARP (2770-3127), pFACMV-MINT and the luciferase reporter construct pGa981/6 (12 x CSL-RE-LUC) were previously described ${ }^{27}$. The expression plasmids for AOS specific mouse RBPJ variants, pcDNA3-FlagRbpj(E89G), pcDNA3-Flag-Rbpj(K195E), pcDNA3-Rbpj-VP16(E89G) and pcDNA3-RbpjVP16(K195E) were made by site directed mutagenesis. All constructs were validated by sequencing.

Bacterial Recombinant Protein Expression and Purification: As previously described ${ }^{24,29}$, competent BL21 Tuner cells transformed with pGEX-6P1-Su(H) or pGEX-6P1-Rbpj were grown at $37^{\circ} \mathrm{C}$ in $\mathrm{LB}+$ ampicillin to an OD of 1.5 followed by overnight IPTG induction at $20^{\circ} \mathrm{C}$. Cell pellets were resuspended in lysis buffer, sonicated, and centrifuged at $15,000 \mathrm{~g} .60 \% \mathrm{w} / \mathrm{v}$ ammonium sulfate was added to the supernatant to precipitate the protein and then centrifuged at $15,000 \mathrm{~g}$. The protein pellet was resuspended in PBS $+0.1 \%$ triton and incubated overnight with glutathione affinity resin. GST-Su(H) or -RBPJ tagged protein was eluted from the column and then cut with PreScission Protease. An SP 
ion exchange column was used to separate the cut GST from the $\mathrm{Su}(\mathrm{H})$ or RBPJ protein, which was then concentrated, flash frozen in liquid nitrogen and stored at $-80^{\circ} \mathrm{C}$. Drosophila Hairless residues 232-358 and human SHARP residues 2776-2833, corresponding to the regions that bind $\mathrm{Su}(\mathrm{H})$ and Rbpj, respectively, were cloned into pSMT3 to produce recombinant protein with an N-terminal SMT3 and His tag and purified as previously described ${ }^{25-27}$.

Peptide Synthesis: $\quad$ Drosophila Notch 1763-1790 (VLSTQRKRAHGVTWFPEGFRAPAAVMSR) and human Notch1 residues 1754-1781 (VLLSRKRRRQHGQLWFPEGFKVSEASKK) corresponding to the fly and mouse RAM domains were chemically synthesized and purified to $95 \%$ purity by Peptide 2.0 .

Electrophoretic Mobility Shift Assays (EMSAs) using Purified Proteins: EMSAs using bacterially purified proteins were performed essentially as previously described ${ }^{39-41}$. In brief, a fluorescent labeled probe containing a high affinity CSL binding site (shown in bold) was generated by annealing an unlabeled 5'

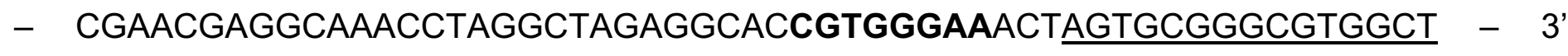
oligonucleotide with a 5'IRDye-700 labeled 5' - AGCCACGCCCGCACT - 3' oligonucleotide that is complementary to the underlined region of the longer oligonucleotide. The Klenow enzyme was used to make the double-stranded probe and the indicated amounts of each $\mathrm{Su}(\mathrm{H})$ and $\mathrm{Rbpj}$ proteins were incubated with $3.4 \mathrm{nM}$ of the labeled probe for 10 minutes at room temperature prior to being loaded onto native acrylamide gel electrophoresis. Acrylamide gels were run at $150 \mathrm{~V}$ for 2 hours and imaged and quantified using the LICOR Odyssey CLx scanner.

In vitro protein translation: The in vitro protein translations were performed using the TNT-assay (L4610) from Promega according to manufacturer's instructions. Prior to EMSAs the in vitro translations of Rbpj (WT) and mutant proteins were monitored by western blotting using an anti-Flag antibody (M5, Merck). 
EMSA from in vitro protein translation system: Reticulocyte lysates from in vitro translations were used for electromobility shift assays (EMSAs) in a binding buffer consisting of $10 \mathrm{mM}$ Tris- $\mathrm{HCl}(\mathrm{pH} 7.5), 100$ $\mathrm{mM} \mathrm{NaCl}, 0.1 \mathrm{mM}$ EDTA, $0.5 \mathrm{mM}$ DTT, and 4\% glycerol. For the binding reaction, $10 \mathrm{ng}(0.02 \mathrm{U})$ poly(dl-dC) (GE healthcare) and approximately $0.5 \mathrm{ng}$ of ${ }^{32} \mathrm{P}$-labeled oligonucleotides were added. The sequence of the double-stranded oligonucleotide with 2 RBPJ binding sites (underlined): 5'-CCT GGA ACT ATT TTC CCA CGG TGC CCT TCC GCC CAT TTT CCC ACG AGT CG-3 and reverse strand: 5'-CTC GCG ACT CGT GGG AAA ATG GGC GGA AGG GCA CCG TGG GAA AAT AGT TC-3'. Super shifting of complexes was achieved by adding $1 \mu \mathrm{g}$ of anti-Flag (M5, Merck) antibody. The reaction products were separated using $5 \%$ polyacrylamide gels with $1 x$ Tris-glycine-EDTA at room temperature. Gels were dried and exposed to X-ray films (GE Healthcare).

Oligonucleotide Preparation for ITC: The following 20-mer oligonucleotide sequences were ordered from Eurofins: 5'-GGCACCGTGGGAAACTAGTG-3' and the reverse compliment 5'CACTAGTTTCCCACGGTGCC-3' showing the Su(H)/RBPJ binding site in bold and underlined. Single stranded oligos were further purified on a Resource $Q$ ion exchange column and then buffer exchanged into an oligo annealing buffer containing $10 \mathrm{mM}$ Tris $8.0,500 \mathrm{mM} \mathrm{NaCl}$, and $1 \mathrm{mM} \mathrm{MgCl}_{2}$. Single stranded oligos were combined in equal molar amounts, boiled for 10 minutes, and then slowly cooled to room temperature to allow for proper annealing.

Isothermal Titration Calorimetry: All proteins, peptides, and oligos were dialyzed overnight in a buffer containing $50 \mathrm{mM}$ sodium phosphate and $150 \mathrm{mM}$ sodium chloride. Experiments were conducted using the VP-ITC MicroCalorimeter manufactured by MicroCal. All protein/DNA binding experiments were performed at $10^{\circ} \mathrm{C}$, while protein/peptide experiments were performed at $20^{\circ} \mathrm{C}$. Each experiment was performed in triplicate with 20 injections, $14 \mu \mathrm{L}$ per injection. Heat of dilution experiments were 
performed by injecting syringe samples into a cell containing only buffer, and all analysis was performed with the heat of dilution subtracted before fitting. The raw data were analyzed using ORIGIN and fit to a one site binding model. A two sample T-test was used to compare WT proteins to each mutant, with a p-value $<0.05$ indicating a significant difference.

Differential Scanning Fluorimetry: $\mathrm{Rbpj} / \mathrm{Su}(\mathrm{H})$ samples were prepared in triplicate at $5 \mu \mathrm{M}$ in buffer containing $50 \mathrm{mM}$ sodium phosphate and $150 \mathrm{mM}$ sodium chloride. DSF was performed by heating samples containing $10 \mathrm{X}$ SYPRO Orange in $1^{\circ} \mathrm{C}$ increments from $25^{\circ} \mathrm{C}$ to $100^{\circ} \mathrm{C}$ using the StepOne real-time PCR machine and using the Protein Thermal Shift software (ThermoFisher) to fit the data.

Genomic DNA Isolation and Sequencing: For the original determination of the mutations in the $S u(H)^{T 4}$ and $\mathrm{Su}(H)^{\mathrm{O} 5}$ alleles, chromosomal DNA was isolated by protease $\mathrm{K}$ digestion of fly lysates. Genomic DNA was subjected to PCR with primers specific to the $\mathrm{Su}(\mathrm{H})$ coding region. Due to the presence of a large intron in the coding region, the first exon was amplified and sequenced separately using two primers TTGCAGCCTTAAACAGAAGCCAGC (forward) and AGCCGGTATTATCAGGTGCTTGGT (reverse). The resulting PCR fragment was sequenced using the same primers as well as an internal primer, ACAAATGCAGATGTCCTTGCTGCC. Exons 2-4 were amplified using two primers, CAAAGCTGCATTGCTTGCGGTT (forward) and TCAATCTACAAACTAAGGTCTTCG (reverse). The resulting PCR fragment was sequenced using these primers as well as two additional primers (ACAGTCAAACTGGTGTGCTCCGTAA and AtgtAgAAgGCGCATTTGTGCAGC). Since the original fly stocks were heterozygous for the $S u(H)^{T 4}$ and $S u(H)^{05}$ alleles, the mutations were identified by the presence of double peaks in the resulting chromatogram. To confirm specific coding variants in each allele prior to our genetic phenotyping assays, genomic DNA was prepared using Qiagen's DNeasy blood purification kit. 10 adult males were used per genotype. DNA was eluted in 100ul Buffer AE. We used two primers (5'-GTCCAGTCCGCAATGAAAAT-3' (forward) and 5'- 
TGCTGCAACATCTCCTCGTA-3' (reverse)) to cover approximately 300bp surrounding each point mutation. The same primers were used for DNA sequencing and ExPasy.org was used to translate nucleotide to amino acid sequence.

Fly Husbandry: All flies were cultured on standard cornmeal food at $25^{\circ} \mathrm{C}$. The following alleles were obtained from the Bloomington Drosophila Stock Center: $\mathrm{Su}(\mathrm{H})^{\top 4}(\# 63234), \mathrm{Su}(\mathrm{H})^{05}(\# 63235)$, $\mathrm{Su}(\mathrm{H})^{\mathrm{IB115}}$ (\#26661), $\mathrm{N}^{55 \mathrm{e} 11}(\# 28813), \mathrm{H}^{1}(\# 515), \mathrm{E}(\mathrm{spl}) \mathrm{m \alpha}-\mathrm{Gal4}, \mathrm{UAS}-\mathrm{GFP}$ (\#26788), PBac\{Su(H)GFP,FLAG\} (\#81279) and PBac\{N-GFP,FLAG\} (\#81271).

Reporter assay in wing disc: Homozygous E(spl)ma-Gal4, UAS-GFP male flies were mated to either WT, Su(H) $)^{\mathrm{IB} 115} /$ cyo,act-GFP, Su(H) ${ }^{\top 4} /$ cyo,act-GFP or $\mathrm{Su}(\mathrm{H})^{\mathrm{O} 5 / c y o, a c t-G F P}$ virginal females. Imaginal wing discs from non-cyo-act-GFP wandering $3^{\text {rd }}$ instar larvae were dissected in PBS and fixed in $4 \%$ formaldehyde for $15 \mathrm{~min}$. Discs were subsequently washed 4 times with PBX (0.3\% TritonX-100 in PBS), and incubated with the primary antibody, anti-Cut (Mouse 1:50, DSHB). No antibody was used for GFP detection. A fluorescent secondary antibody (Goat anti-Mouse 555 Alexa Fluor, Molecular Probes) was used to detect the Cut-positive wing margin cells. For quantitative purposes, all imaginal discs were dissected, fixed, and imaged at the same time on a Nikon A1R inverted confocal microscope (40x objective) with identical exposure settings. Z-stack images were analyzed using Imaris software. A Student's t-test was used to determine significance.

Genetic assays: We analyzed the wing nicking and macrochaetae phenotypes essentially as previously described $^{39}$. In brief, flies of the appropriate genotypes were mated and transferred to fresh food every other day to avoid overcrowding. Offspring of the listed genotypes were selected and the number of nicks on each wing and/or the number of macrochaetae on the dorsal head and thorax was recorded. Proportional odds model with Bonferonni adjustment and two-sided Fisher's exact test were used to 
determine significance for wing nicking. Student's t-test and two-sided Fisher's exact test were used to determine significance for bristle number.

Cell culture and preparation of cell extracts: Rbpj-depleted hybridoma mature T (MT) cell line was grown in Iscove's Modified Dulbecco Medium (IMDM, Gibco) supplemented with 2\% FCS, $0.3 \mathrm{mg} / \mathrm{l}$ peptone, $5 \mathrm{mg} / \mathrm{l}$ insulin, nonessential aminoacids and penicillin/streptomycin. Cells were grown at $37^{\circ} \mathrm{C}$ with $5 \%$ $\mathrm{CO}_{2}$. Phoenix ${ }^{\mathrm{TM}}$ packaging cells (Orbigen, Inc. San Diego, CA, USA) were cultivated in Dulbecco's Modified Eagle Medium (DMEM, Gibco) supplemented with $10 \%$ fetal calf serum (FCS) and penicillin/streptomycin. CRISPR/Cas9-mediated Rbpj depleted hybridoma mature $\mathrm{T}$ cells were generated as previously described ${ }^{27}$. Rbpj-depleted mature T-cell lines stably expressing Rbpj ${ }^{\mathrm{WT}}$, Adams-Oliver mutant Rbpje89G or Adams-Oliver mutant Rbpj ${ }^{\mathrm{K} 195 \mathrm{E}}$ were generated as follows: $5 \times 10^{6}$ Phoenix ${ }^{\mathrm{TM}}$ cells were transfected with $20 \mu \mathrm{g}$ of the retroviral plasmid DNA mixed with $860 \mu \mathrm{l}$ of $\mathrm{H}_{2} \mathrm{O}$ and $120 \mu$ of $2 x$ HBS buffer (50 mM HEPES pH 7.05, $10 \mathrm{mM} \mathrm{KCl,} 12 \mathrm{mM}$ Glucose, $280 \mathrm{mM} \mathrm{NaCl}, 1.5 \mathrm{mM}$ $\mathrm{NaHPO}_{4}$ ) while vortexing and the solution was incubated $20 \mathrm{~min}$ at room temperature. In the meantime, $25 \mu \mathrm{M}$ Chloroquine solution (Sigma-Aldrich) was added to the Phoenix ${ }^{\mathrm{TM}}$ cells (1 microliter/ml) and the cells were incubated for $10 \mathrm{~min}$. The DNA solution was added to the cells and $12 \mathrm{~h}$ later the medium was replaced. After $24 \mathrm{~h}$ of incubation, the medium containing the retroviral suspension was filtered and Polybrene (Sigma-Aldrich) solution was added. Fresh medium was added to the Phoenix ${ }^{\mathrm{TM}}$ cells that were maintained in culture for further infections. The retroviral solution was used for spin infection of Rbpj-depleted MT cells by centrifuging $45 \mathrm{~min}$ at $1800 \mathrm{rpm}$ at $37^{\circ} \mathrm{C}$. In total, four spin infections were performed over two days. Positively infected cells were selected with Blasticidin (Gibco).

Preparation of protein extracts and western blotting from Rbpj-depleted mature $T$ cells: The nuclear extract (NE) from MT cells overexpressing the Rbpj constructs or control cells containing empty vector was prepared as follows. Briefly, $10 \times 10^{6}$ cells were washed with PBS and resuspended in $200 \mu \mathrm{l}$ of Buffer 1 (10 mM HEPES pH 7.9, $10 \mathrm{mM} \mathrm{KCl,} 0.1 \mathrm{mM}$ EDTA, $0.1 \mathrm{mM}$ EGTA, 1mM beta- 
mercaptoethanol, supplemented with PMSF). The cell suspension was incubated 10 min on ice, $5 \mu$ of $10 \%$ NP-40 were added and mixed by vortexing. After $10 \mathrm{~s}$ of centrifugation at $13000 \mathrm{rpm}$ at $4^{\circ} \mathrm{C}$, the nuclei pellet was washed twice in $500 \mu$ of Buffer 1 and resuspended in $100 \mu$ of Buffer 2 (20 mM HEPES pH 7.9, 400 mM NaCl, 1 mM EDTA, 1 mM EGTA, 1 mM beta-mercaptoethanol, supplemented with PMSF). After 20 min of incubation on ice, the nuclei suspension was centrifuged 10 min at 13000 $\mathrm{rpm}$ at $4^{\circ} \mathrm{C}$. and the supernatant was collected for further analysis. Protein concentration was measured by Bradford assay (Biorad) and samples were boiled after adding SDS-polacrylamide gel loading buffer. Samples were resolved by SDS-Page and analyzed by Western blotting using antibodies against RBPJ (Cosmo Bio Co. LTD) or TBP (Santa Cruz Biotechnology).

Gene expression analysis as measured by qRT-PCR in hybridoma mature T-cells: Total RNA was purified using TRIzol reagent accordingly to manufacturer's instructions. $1 \mu \mathrm{g}$ of RNA was reverse transcribed in cDNA using random hexamers and M-MuLV reverse transcriptase (New England Biolabs). Quantitative PCRs were assembled with Absolute QPCR ROX Mix (Thermo Scientific, AB1139), gene-specific oligonucleotides and double-dye probes and analyzed using StepOne Plus Real Time PCR system (Applied Biosystem). Data were normalized to the housekeeping gene Beta actin (Bact) and represent the mean \pm SD of three independent experiments $\left({ }^{* * *} p<0.001 ;{ }^{* *} p<0.01,{ }^{*} p<0.05\right.$ and [ns], not significant, unpaired Student's t-test).

Coimmunoprecipitation experiments: HEK293 and HeLa cells were transfected using the Profectin and Lipofectamine 2000 transfection reagent, respectively, according to the manufacturer's instructions. HEK293 cells were transfected with the indicated constructs for expression of untagged, GFP- and Flag-tagged WT and mutant proteins. 24 hours after transfection cells were lysed with $600 \mu$ l CHAPS lysis buffer [10 mM 3-[(3-Cholamidopropyl)dimethylammonio]-1-propanesulfonate hydrate (CHAPS, Roth), 50 mM Tris- $\mathrm{HCl}$ (pH 7.8), 150 mM NaCl, 5 mM NaF, 1 mM Dithiothreitol (DTT, Merck), 0.5 mM Phenylmethanesulfonyl fluoride (PMSF, Merck) and $40 \mu \mathrm{l} / \mathrm{ml}$ "Complete Mix" protease inhibitor cocktail 
(Roche)]. The extracts were incubated with $40 \mu \mathrm{l}$ agarose-conjugated anti-Flag antibody (M2, Sigma) at $4^{\circ} \mathrm{C}$ overnight. Precipitates were washed 6 to 8 times with CHAPS lysis buffer and finally resuspended in SDS-polyacrylamide gel loading buffer. For western blotting the proteins were resolved in SDS-polyacrylamide gels and transferred electrophoretically at room temperature to PVDF membranes (Merck) for $1 \mathrm{~h}$ at $50 \mathrm{~mA}$ using a Tris-glycine buffer system. The membranes were preblocked for $1 \mathrm{~h}$ in a solution of $3 \%$ milk powder in PBS-T $(0.1 \%$ Tween 20 in PBS) before adding antibodies. The following antibodies were used: anti-GFP (7.1/13.1, mouse monoclonal IgG, secondary antibody peroxidase conjugated sheep anti-mouse IgG, NA931V, GE healthcare) or anti-Flag (M5, Sigma; secondary antibody, NA931V, GE healthcare). Anti-Mam1 (ab155786), rabbit polyclonal, Abcam, (1:1000), overnight, $4^{\circ} \mathrm{C}$, secondary: HRP-linked-donkey-anti-rabbit IgG (NA934V), GEhealthcare, (1:5000), $1 \mathrm{~h}$, rt.

Fluorescence Microscopy: HeLa cells were cultured on glass coverslips at a density of $10^{5}$ cells per $\mathrm{cm}^{2}$. After $16 \mathrm{~h}$ cells were transfected with $300 \mathrm{ng}$ of expression plasmids using the Lipofectamine 2000 transfection reagent. $24 \mathrm{~h}$ after transfection cells were rinsed with PBS, fixed with $4 \%$ paraformaldehyde (PFA, Merck) in PBS ( $\mathrm{pH}=7.5)$. Specimens were embedded in "ProLong@ Gold antifade" reagent (Thermofisher) supplemented with 2-(4-carbamimidoylphenyl)-1H-indol-6-carboximidamide (DAPI) and stored at $4^{\circ} \mathrm{C}$ overnight. Pictures were taken using a fluorescence microscope (IX71, Olympus) equipped with a digital camera (C4742, Hamamatsu), and a 100-W mercury lamp (HBO 103W/2, Osram). The following filter sets were used: Green, (EGFP) ex: HQ470/40, em: HQ525/50, blue (DAPI) D360/50, em: D460/50.

Luciferase Assays: HeLa cells and HeLa ${ }^{\text {RBPJ-KO }}$ cells were seeded in 48-well plates at a density of $2 \times$ $10^{5}$ cells. Transfection was performed with Lipofectamine 2000 reagent as described above using $1 \mu \mathrm{g}$ of reporter plasmid alone or together with various amount of expression plasmids (given in the 
corresponding figure legends). After $24 \mathrm{~h}$ luciferase activity was determined from at least four independent experiments with $20 \mu \mathrm{l}$ of cleared lysate in an LB 9501 luminometer (Berthold) by using the luciferase assay system from Promega.

qRT-PCR: Total RNA was purified using Trizol reagent (Ambion, 15596018) accordingly to manufacturer's instructions. $1 \mu \mathrm{g}$ of RNA was reverse transcribed in cDNA using random hexamers and M-MuLV reverse transcriptase (NEB). qPCRs were assembled with Absolute QPCR ROX Mix (Thermo Scientific, AB-1139), gene-specific oligonucleotides and double-dye probes and analyzed using the StepOne Plus Real Time PCR system (Applied Biosystem). Data were normalized to the housekeeping gene Bact.

Cyclohexamide Assays: Mouse mK4 cell line was maintained in DMEM/F12 media supplemented with 10\%FBS, penicillin, and streptomycin. Transfection of 6xMyc-tagged RBPJ constructs (pCS2-RBPJ6xMyc, WT and AOS variants) was carried out with TransIT-293 transfection reagent (Mirus) following the manufacture's instruction. After $24 \mathrm{~h}$ of the transfection, the cells were treated with cycloheximide (500ng/ml) and harvested at 48, 72, and $96 \mathrm{~h}$. Protein samples were subjected to SDS-PAGE and subsequently transferred to PVDF membranes. The following primary antibodies were used at 1:1000 dilution in PBST (0.05\% Tween-20) and 5\% non-fat dry milk and incubated overnight at $4^{\circ} \mathrm{C}: \mathrm{Myc}(\mathrm{Cell}$ Signaling Technology \#2276), RBPJ (Cell Signaling Technology \#5313), and actin (Cell Signaling Technology \#5125). Protein bands were visualized by SuperSignal West Pico PLUS enhanced chemiluminescent reagent (ThermoFisher) and detected by ChemiDoc MP system (BioRad). 


\section{REFERENCES}

$1 \quad$ Kovall, R. A., Gebelein, B., Sprinzak, D. \& Kopan, R. The Canonical Notch Signaling Pathway: Structural and Biochemical Insights into Shape, Sugar, and Force. Dev Cell 41, 228-241, doi:10.1016/j.devcel.2017.04.001 (2017).

2 Bray, S. J. Notch signalling in context. Nat Rev Mol Cell Biol 17, 722-735, doi:10.1038/nrm.2016.94 (2016).

3 Hori, K., Sen, A. \& Artavanis-Tsakonas, S. Notch signaling at a glance. J Cell Sci 126, 2135-2140, doi:10.1242/jcs.127308 (2013).

4 Siebel, C. \& Lendahl, U. Notch Signaling in Development, Tissue Homeostasis, and Disease. Physiological reviews 97, 1235-1294, doi:10.1152/physrev.00005.2017 (2017).

5 Kopan, R. \& Ilagan, M. X. The canonical Notch signaling pathway: unfolding the activation mechanism. Cell 137, 216-233, doi:10.1016/j.cell.2009.03.045 (2009).

6 Musse, A. A., Meloty-Kapella, L. \& Weinmaster, G. Notch ligand endocytosis: mechanistic basis of signaling activity. Semin Cell Dev Biol 23, 429-436, doi:10.1016/j.semcdb.2012.01.011 (2012).

7 Kovall, R. A. \& Blacklow, S. C. Mechanistic insights into Notch receptor signaling from structural and biochemical studies. Curr Top Dev Biol 92, 31-71, doi:S0070-2153(10)92002-4 [pii]

10.1016/S0070-2153(10)92002-4 (2010).

8 Bray, S. \& Bernard, F. Notch targets and their regulation. Curr Top Dev Biol 92, 253-275, doi:S00702153(10)92008-5 [pii]

10.1016/S0070-2153(10)92008-5 (2010).

9 Hall, D. P. \& Kovall, R. A. Structurally conserved binding motifs of transcriptional regulators to notch nuclear effector CSL. Exp Biol Med (Maywood), 1535370219877818, doi:10.1177/1535370219877818 (2019).

10 Oswald, F. \& Kovall, R. A. CSL-Associated Corepressor and Coactivator Complexes. Adv Exp Med Biol 1066, 279-295, doi:10.1007/978-3-319-89512-3_14 (2018).

11 Maier, D. Hairless: the ignored antagonist of the Notch signalling pathway. Hereditas 143, 212-221, doi:10.1111/j.2007.0018-0661.01971.x (2006).

12 Masek, J. \& Andersson, E. R. The developmental biology of genetic Notch disorders. Development 144, 1743-1763, doi:10.1242/dev.148007 (2017).

13 Gilbert, M. A. \& Spinner, N. B. Alagille syndrome: Genetics and Functional Models. Curr Pathobiol Rep 5, 233-241, doi:10.1007/s40139-017-0144-8 (2017).

14 Hassed, S. J. et al. RBPJ mutations identified in two families affected by Adams-Oliver syndrome. Am J Hum Genet 91, 391-395, doi:10.1016/j.ajhg.2012.07.005 (2012).

15 Fortini, M. E. \& Artavanis-Tsakonas, S. The suppressor of hairless protein participates in notch receptor signaling. Cell 79, 273-282, doi:10.1016/0092-8674(94)90196-1 (1994).

16 Schweisguth, F. \& Posakony, J. W. Antagonistic activities of Suppressor of Hairless and Hairless control alternative cell fates in the Drosophila adult epidermis. Development 120, 1433-1441 (1994).

17 Micchelli, C. A., Rulifson, E. J. \& Blair, S. S. The function and regulation of cut expression on the wing margin of Drosophila: Notch, Wingless and a dominant negative role for Delta and Serrate. Development 124, 1485-1495 (1997).

18 Neumann, C. J. \& Cohen, S. M. A hierarchy of cross-regulation involving Notch, wingless, vestigial and cut organizes the dorsal/ventral axis of the Drosophila wing. Development 122, 3477-3485 (1996).

19 de Celis, J. F., Garcia-Bellido, A. \& Bray, S. J. Activation and function of Notch at the dorsal-ventral boundary of the wing imaginal disc. Development 122, 359-369 (1996).

20 Zacharioudaki, E. \& Bray, S. J. Tools and methods for studying Notch signaling in Drosophila melanogaster. Methods 68, 173-182, doi:10.1016/j.ymeth.2014.03.029 (2014).

21 Lai, E. C., Bodner, R. \& Posakony, J. W. The enhancer of split complex of Drosophila includes four Notch-regulated members of the bearded gene family. Development 127, 3441-3455 (2000).

22 Schweisguth, F. \& Posakony, J. W. Suppressor of Hairless, the Drosophila homolog of the mouse recombination signal-binding protein gene, controls sensory organ cell fates. Cell 69, 1199-1212, doi:10.1016/0092-8674(92)90641-o (1992). 
23 Furukawa, T., Maruyama, S., Kawaichi, M. \& Honjo, T. The Drosophila homolog of the immunoglobulin recombination signal-binding protein regulates peripheral nervous system development. Cell 69, 11911197, doi:10.1016/0092-8674(92)90640-x (1992).

24 Contreras, A. N., Yuan, Z. \& Kovall, R. A. Thermodynamic binding analysis of Notch transcription complexes from Drosophila melanogaster. Protein Sci 24, 812-822, doi:10.1002/pro.2652 (2015).

25 Yuan, Z. et al. Structure and Function of the $\mathrm{Su}(\mathrm{H})$-Hairless Repressor Complex, the Major Antagonist of Notch Signaling in Drosophila melanogaster. PLoS Biol 14, e1002509, doi:10.1371/journal.pbio. 1002509 (2016).

26 Maier, D. et al. Structural and functional analysis of the repressor complex in the Notch signaling pathway of Drosophila melanogaster. Mol Biol Cell 22, 3242-3252, doi:mbc.E11-05-0420 [pii]

10.1091/mbc.E11-05-0420 (2011).

27 Yuan, Z. et al. Structural and Functional Studies of the RBPJ-SHARP Complex Reveal a Conserved Corepressor Binding Site. Cell reports 26, 845-854 e846, doi:10.1016/j.celrep.2018.12.097 (2019).

28 VanderWielen, B. D., Yuan, Z., Friedmann, D. R. \& Kovall, R. A. Transcriptional repression in the Notch pathway: thermodynamic characterization of CSL-MINT (Msx2-interacting nuclear target protein) complexes. J Biol Chem 286, 14892-14902, doi:10.1074/jbc.M110.181156 (2011).

29 Friedmann, D. R., Wilson, J. J. \& Kovall, R. A. RAM-induced allostery facilitates assembly of a notch pathway active transcription complex. $J$ Biol Chem 283, 14781-14791, doi:M709501200 [pii]

10.1074/jbc.M709501200 (2008).

30 Wolf, D. et al. Nucleo-cytoplasmic shuttling of Drosophila Hairless/Su(H) heterodimer as a means of regulating Notch dependent transcription. Biochim Biophys Acta Mol Cell Res 1866, 1520-1532, doi:10.1016/j.bbamcr.2019.07.008 (2019).

31 Hassed, S., Li, S., Mulvihill, J., Aston, C. \& Palmer, S. Adams-Oliver syndrome review of the literature: Refining the diagnostic phenotype. Am J Med Genet A 173, 790-800, doi:10.1002/ajmg.a.37889 (2017).

32 Meester, J. A. N. et al. Elucidating the genetic architecture of Adams-Oliver syndrome in a large European cohort. Hum Mutat 39, 1246-1261, doi:10.1002/humu.23567 (2018).

33 Frankenreiter, L. et al. Phospho-Site Mutations in Transcription Factor Suppressor of Hairless Impact Notch Signaling Activity During Hematopoiesis in Drosophila. Front Cell Dev Biol 9, 658820, doi:10.3389/fcell.2021.658820 (2021).

34 Nagel, A. C. et al. Phosphorylation of Suppressor of Hairless impedes its DNA-binding activity. Scientific reports 7, 11820, doi:10.1038/s41598-017-11952-0 (2017).

35 Yuan, Z., Friedmann, D. R., VanderWielen, B. D., Collins, K. J. \& Kovall, R. A. Characterization of CSL (CBF-1, Su(H), Lag-1) mutants reveals differences in signaling mediated by Notch1 and Notch2. J Biol Chem 287, 34904-34916, doi:M112.403287 [pii]

10.1074/jbc.M112.403287 (2012).

36 Lubman, O. Y., Ilagan, M. X., Kopan, R. \& Barrick, D. Quantitative dissection of the Notch:CSL interaction: insights into the Notch-mediated transcriptional switch. J Mol Biol 365, 577-589, doi:10.1016/j.jmb.2006.09.071 (2007).

37 Friedmann, D. R. \& Kovall, R. A. Thermodynamic and structural insights into CSL-DNA complexes. Protein Sci 19, 34-46, doi:10.1002/pro.280 (2010).

38 Choi, S. H. et al. Conformational locking upon cooperative assembly of notch transcription complexes. Structure 20, 340-349, doi:10.1016/j.str.2011.12.011 (2012).

39 Kuang, Y. et al. Enhancer architecture sensitizes cell specific responses to Notch gene dose via a bind and discard mechanism. eLife 9, doi:10.7554/eLife.53659 (2020).

40 Uhl, J. D., Zandvakili, A. \& Gebelein, B. A Hox Transcription Factor Collective Binds a Highly Conserved Distal-less cis-Regulatory Module to Generate Robust Transcriptional Outcomes. PLoS genetics 12, e1005981, doi:10.1371/journal.pgen.1005981 (2016).

41 Uhl, J. D., Cook, T. A. \& Gebelein, B. Comparing anterior and posterior Hox complex formation reveals guidelines for predicting cis-regulatory elements. Developmental biology 343, 154-166, doi:10.1016/j.ydbio.2010.04.004 (2010). 
A
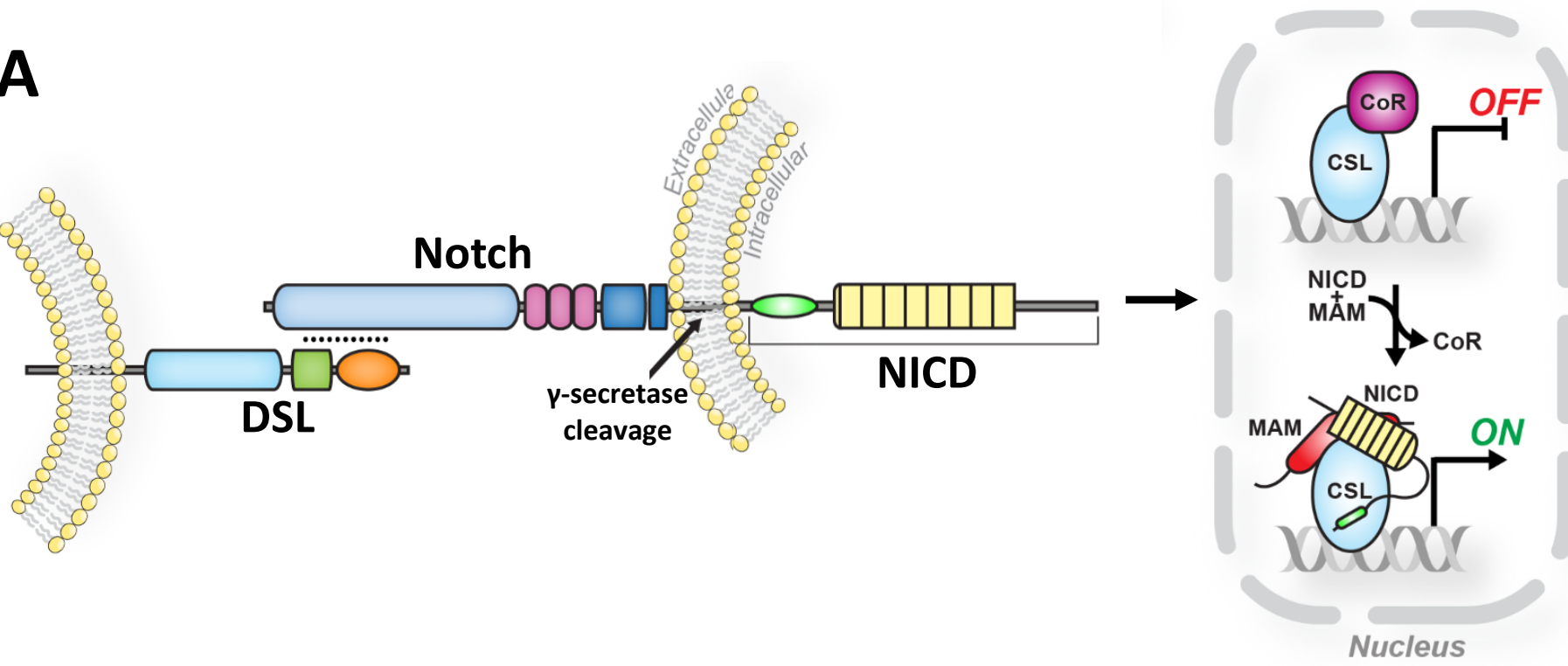

B

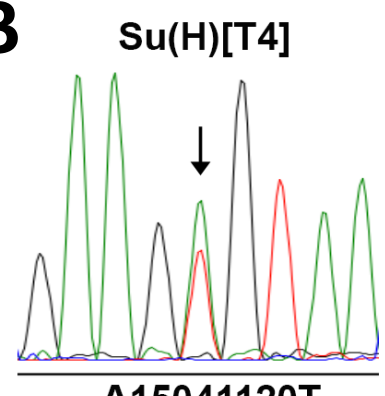

A15041120T

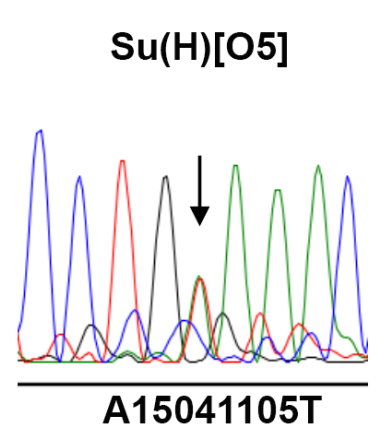

C

D

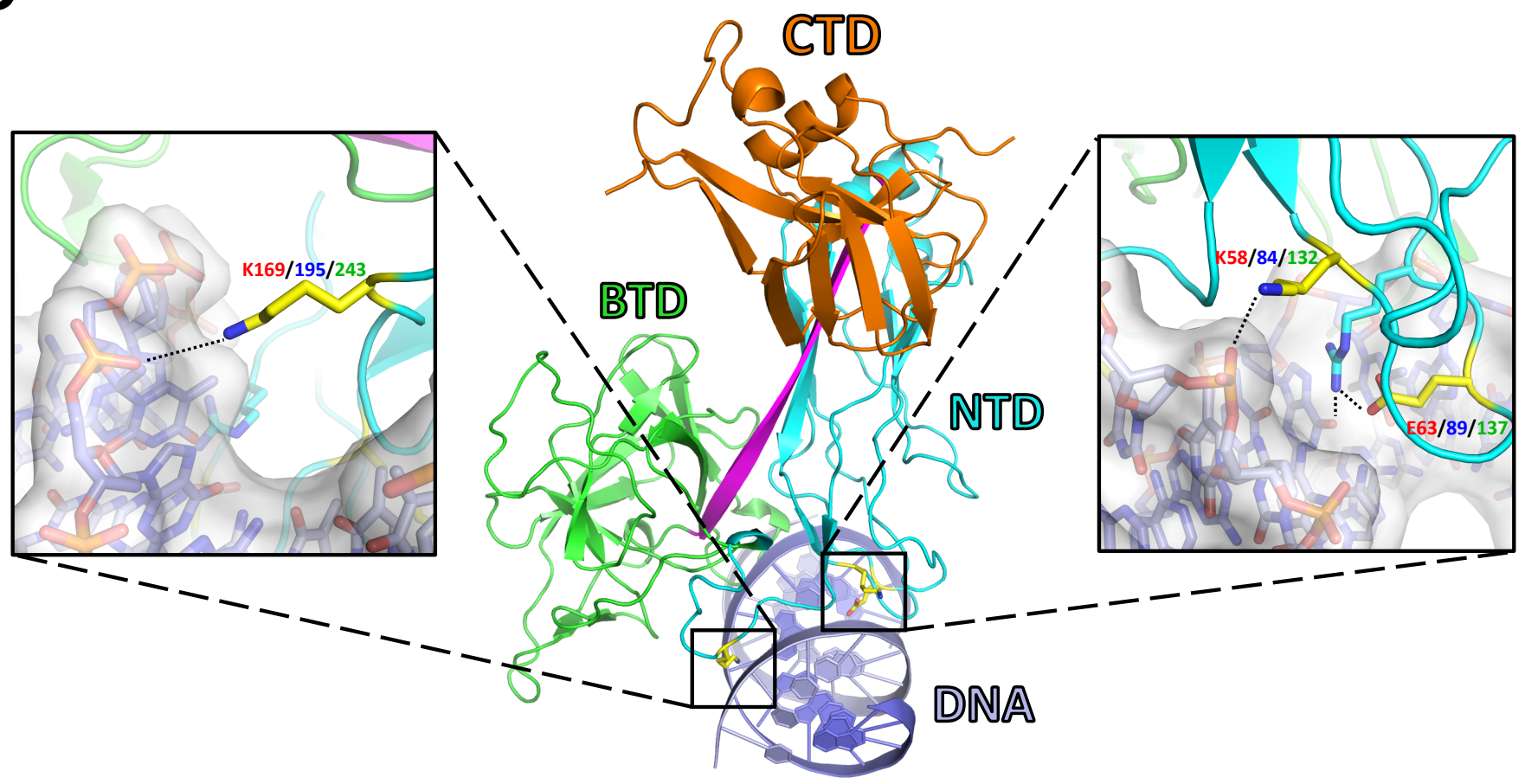

Fig 1 
A

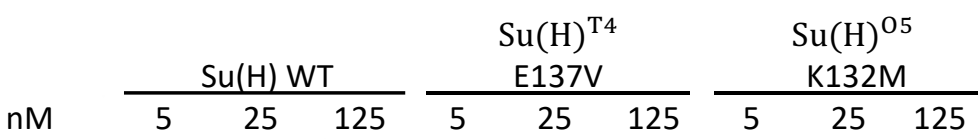
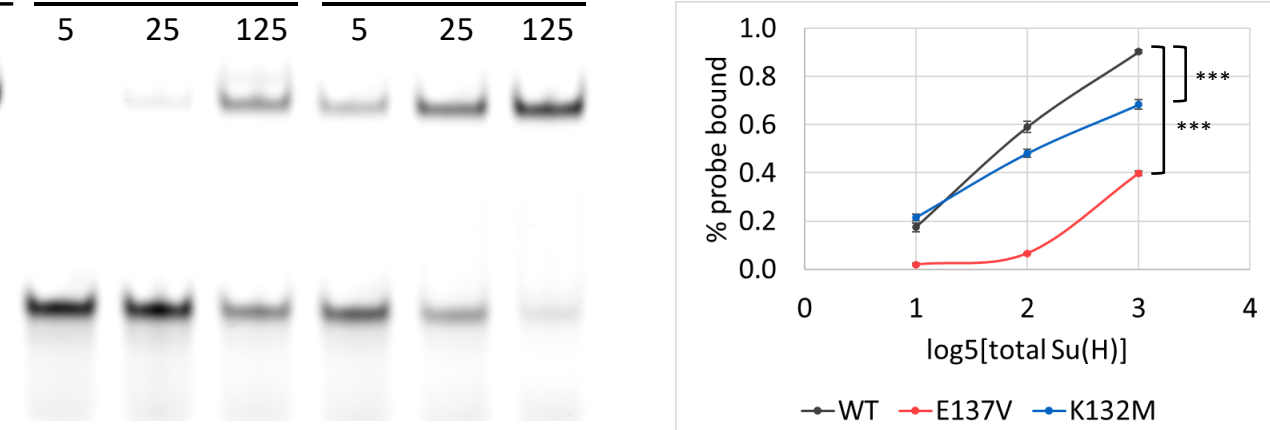

B
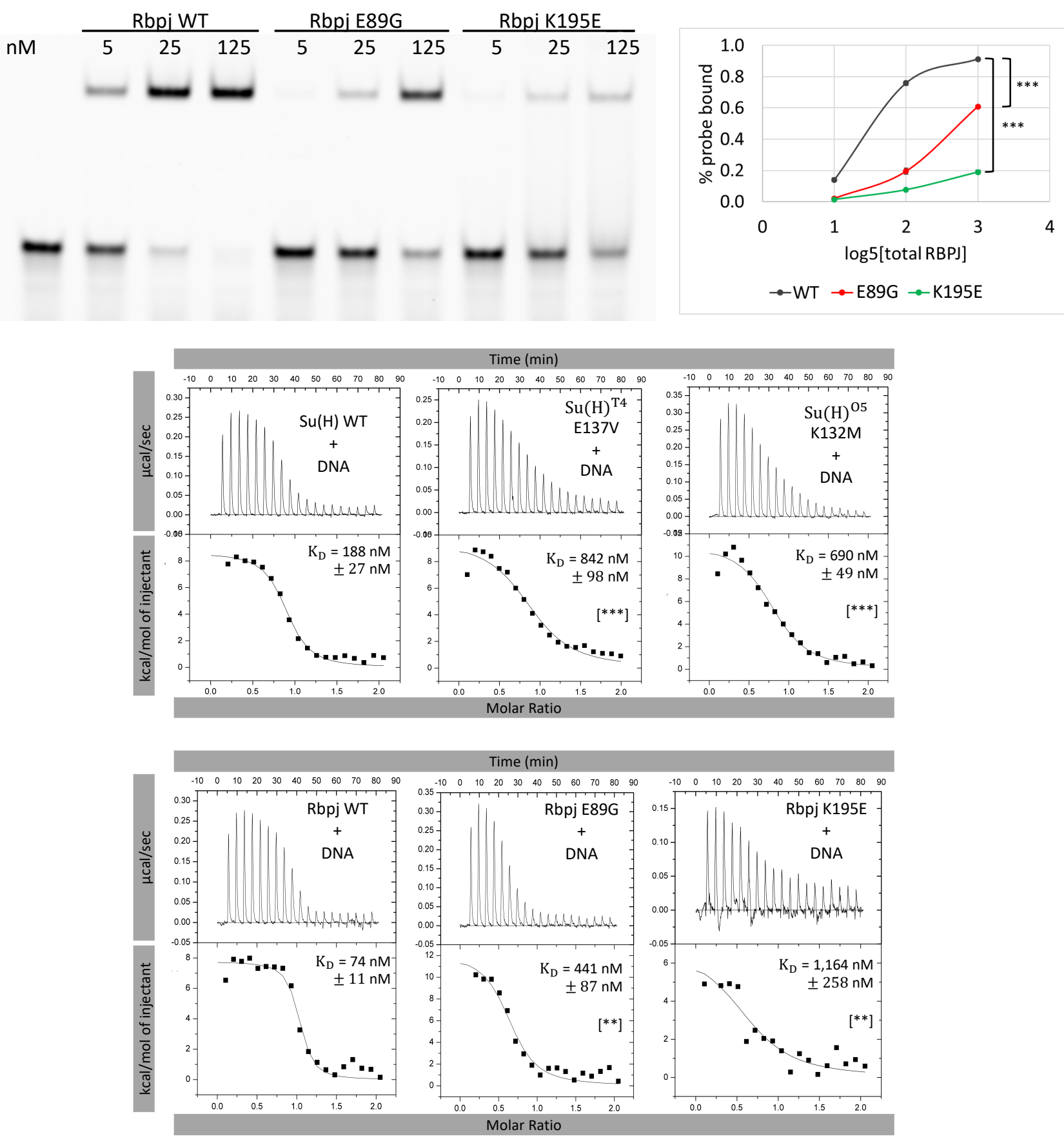

Fig 2 
A

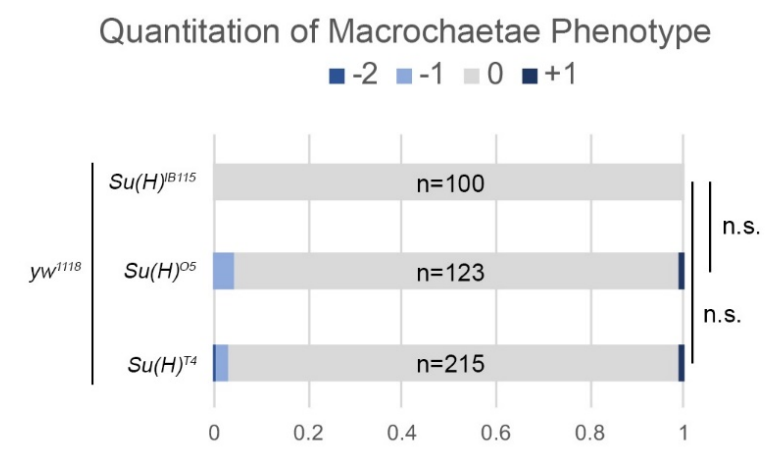

B

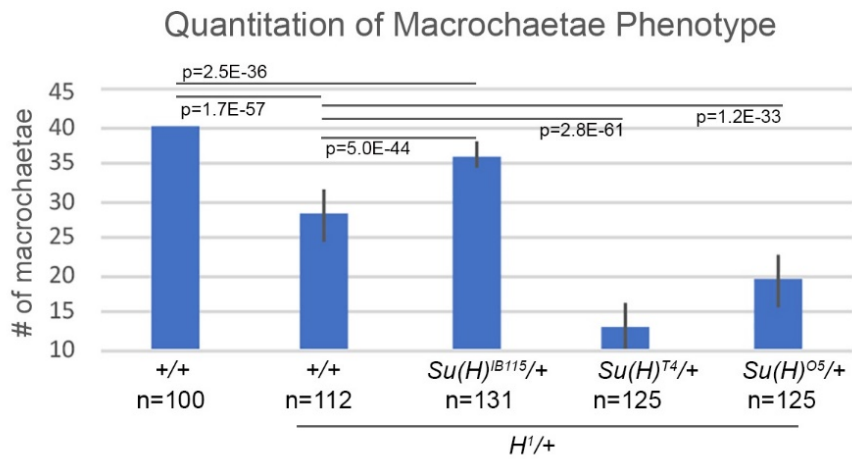

G

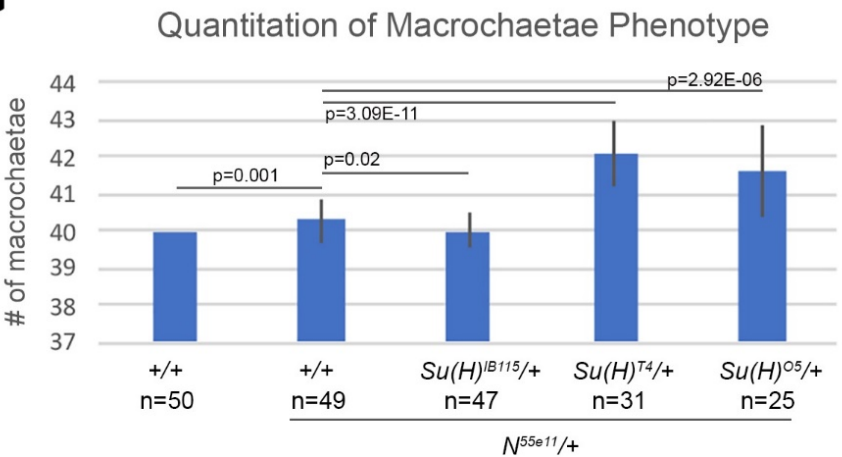

$\mathrm{H}$

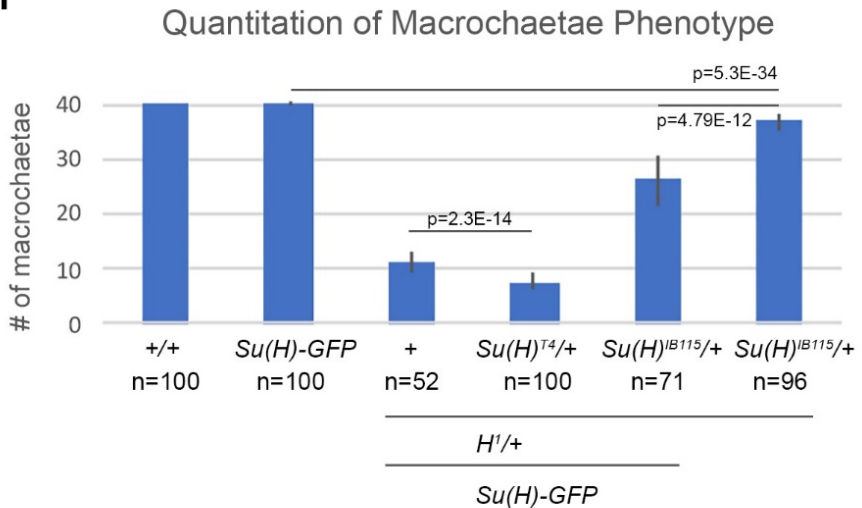

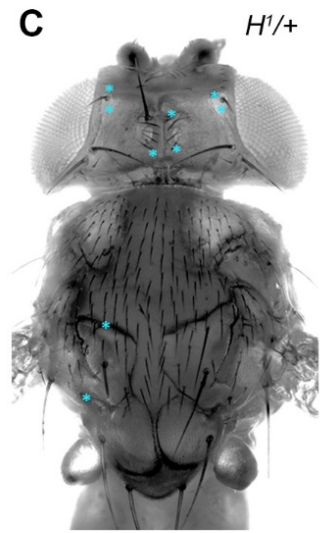

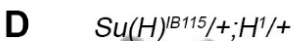

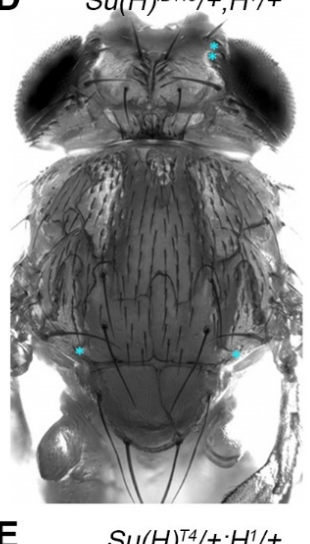

E $\mathrm{Su}(H)^{\top 4 /+} ; H^{1 /+}$

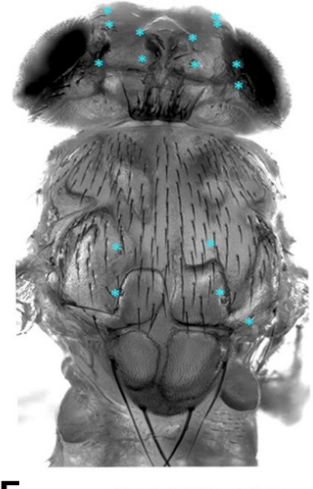

F $\mathrm{Su}(H)^{05 /+} ; H^{1 /+}$

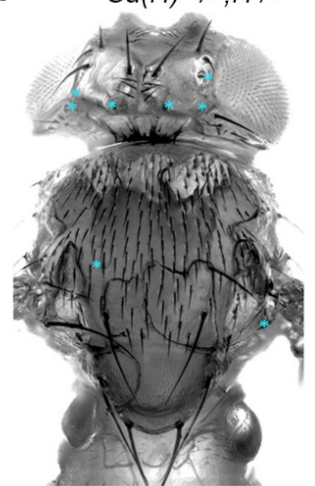


A

B
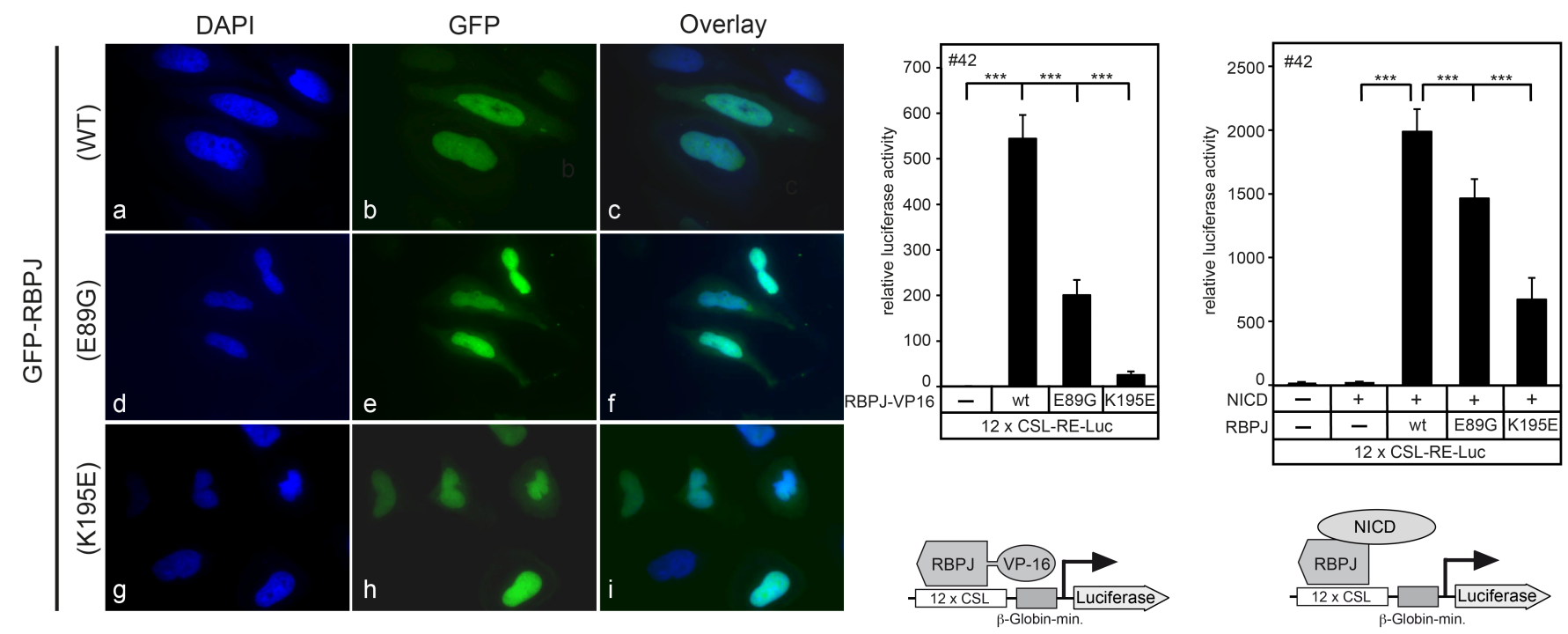

D
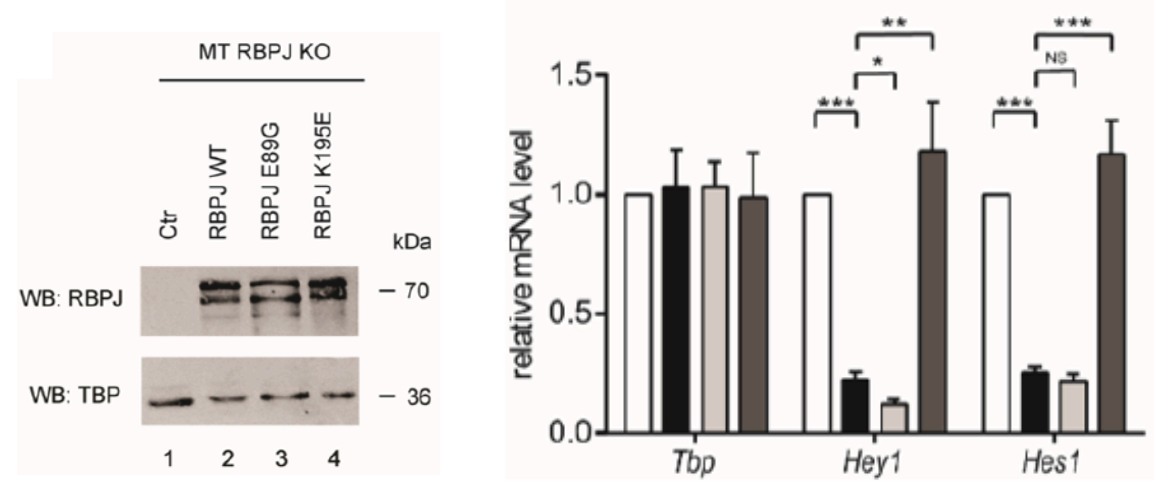

$\square$ MT RBPJ KO + Ctr

- MT RBPJ KO + RBPJ WT

$\square$ MT RBPJ KO + RBPJ E89G

$\square$ MT RBPJ KO + RBPJ K195E 